\title{
Global Existence and Asymptotic Behavior of Solutions for the Maxwell-Schrödinger Equations in Three Space Dimensions
}

\author{
Yoshio Tsutsumi \\ Department of Mathematical Sciences, University of Tokyo, Hongo, Tokyo 113, Japan \\ Received March 19, 1992; in revised form July 15, 1992
}

Dedicated to Professor R. Iino on his 70th birthday

\begin{abstract}
In this paper we study the global existence and asymptotic behavior of solutions for the Maxwell-Schrödinger equations under the Coulomb gauge condition in three space dimensions with the final states given at $t=+\infty$. This leads to the construction of the modified wave operator for certain scattered data. It is also shown that for the initial data in the range of the modified wave operator, the initial value problem of the Maxwell-Schrödinger equations has the global solutions in time.
\end{abstract}

\section{Introduction and Main Results}

In the present paper we consider the global existence and asymptotic behavior of solutions for the Maxwell-Schrödinger equations under the Coulomb gauge condition in three space dimensions:

$$
\begin{gathered}
\frac{\partial^{2}}{\partial t^{2}} A-\Delta A=-i\{\bar{\psi}(\nabla-i A) \psi-\psi(\nabla+i A) \bar{\psi}\} \\
-\frac{1}{2 \pi} \frac{\partial}{\partial t} \nabla\left(\frac{1}{|x|} *|\psi|^{2}\right), \quad t>0, x \in \mathbf{R}^{3}, \\
2 i \frac{\partial}{\partial t} \psi+(\nabla-i A)^{2} \psi-\frac{1}{4 \pi}\left(\frac{1}{|x|} *|\psi|^{2}\right) \psi=0, \quad t>0, x \in \mathbf{R}^{3}, \\
\operatorname{div} A=0, \quad t \geqq 0, x \in \mathbf{R}^{3},
\end{gathered}
$$

where $*$ denotes the convolution with respect to the spatial variables. Here, $A(t, x)$ is a function from $[0, \infty) \times \mathbf{R}^{3}$ to $\mathbf{R}^{3}$ which denotes the electromagnetic real vector potential, and $\psi(t, x)$ is a function from $[0, \infty) \times \mathbf{R}^{3}$ to $\mathbf{C}$ which denotes the complex scalar field of nonrelativistic charged particles. Equations (1.1)-(1.3) are the classical approximation to the quantum field equations for an electrodynamical nonrelativistic many body system. 
In $[11,12]$, Nakamitsu and M. Tsutsumi studied the solvability of the initial value problem of the Maxwell-Schrödinger equations under the Lorentz gauge condition, instead of the Coulomb gauge condition. In $[11,12]$, it is proved that if the spatial dimensions are two or one, the initial value problem of the Maxwell-Schrödinger equations with the Lorentz gauge condition is globally solvable and that if the spatial dimensions are three, the initial value problem with the Lorentz gauge condition is locally solvable. However, there seem to be no results concerning the global existence and asymptotic behavior of solutions for (1.1)-(1.2) in three space dimensions until now. In the present paper, instead of the initial value problem we consider solving (1.1)-(1.3) with the final states given at $t=+\infty$. The difficulty of constructing the global solutions of (1.1)-(1.3) consists in the quadratic nonlinearity of (1.1)-(1.2). In [10] Klainerman introduced the notion of the null condition to show the global existence of small amplitude solutions for the wave equation with quadratic nonlinear term. Recently, Bachelot [1] and Georgiev [5] have improved the null condition technique to show the global existence of small amplitude solutions for the Dirac-Klein-Gordon equations and the Maxwell-Dirac equations, respectively, which are the classical field equations related to (1.1)-(1.3). However, the null condition technique does not seem to be directly applicable to (1.1)-(1.3), because the null condition technique is based on the Lorentz invariance of the equations. But the Schrödinger equation does not necessarily have the same invariance as the wave equation, and especially the Schrödinger equation is not invariant under the Lorentz transform.

While the initial value problem is considered in [1, 5 and 10], in [3] Flato, Simon and Taflin study the global existence and asymptotic behavior of solutions for the Maxwell-Dirac equations with the final states given at $t=+\infty$. This corresponds to the construction of the wave operator. But in fact, the wave operator cannot be constructed in the usual sense, because the phase of asymptotic profile is distorted by the long range effect arising from the quadratic nonlinearity of the Maxwell-Dirac equations. In [3] they take the phase shift of asymtotic profile into account in order to compare the asymptotic behavior of the MaxwellDirac system with that of the free dynamics (see also [5]). This corresponds to the construction of the so-called modified wave operator. In [3] the phase function indicating the phase shift of asymptotic profile is given as an approximate solution of the classical Hamilton-Jacobi equation associated with a relativistic electron in an external field. Recently, in [13] Ozawa has given a more explicit form of the phase function for the one dimensional Schrödinger equation with power nonlinearity of long range effect. In [8] Hayashi and Ozawa have also shown the existence of the modified wave operator for the derivative nonlinear Schrödinger equation, following [13]. In this paper we shall give an explicit form of the phase function indicating the phase shift of asymptotic profile and construct the modified wave operator of the Maxwell-Schrödinger equations for certain scattered data. We shall first eliminate the long range effect of the worst term by using the difference between the propagation properties of the Maxwell wave and the Schrödinger wave, following [14] and we shall next construct the phase function of the phase shift resulting from the long range effect of the rest term in a similar way to [13] and [8]. Since the long range effect of the Maxwell-Schrödinger equations is more complicated than that of the decoupled nonlinear Schrödinger equation, our proof is more complicated and delicate than that of [13] or [8].

Before we state the main results in this paper, we give several notations. For $1 \leqq p \leqq+\infty$, we denote the standard $L^{p}$ space on $\mathbf{R}^{3}$ by $L^{p}$. For $m, s \in \mathbf{R}$, let $H^{m}$ 
and $H^{m, s}$ be the Banach spaces defined by the completion of the Schwartz space $\mathscr{S}\left(\mathbf{R}^{3}\right)$ in the norms

$$
\begin{aligned}
\|v\|_{H^{m}} & =\left\|(1-\Delta)^{m / 2} v\right\|_{L^{2}}, \\
\|v\|_{H^{m, s}} & =\left\|\left(1+|x|^{2}\right)^{s / 2}(1-\Delta)^{m / 2} v\right\|_{L^{2}},
\end{aligned}
$$

respectively. For $m>0$, let $\dot{H}^{m}$ be the completion of $\mathscr{S}\left(\mathbf{R}^{3}\right)$ with respect to the semi-norm

$$
\|v\|_{\dot{H}^{m}}=\left\|(-\Delta)^{m / 2} v\right\|_{L^{2}}
$$

For $1 \leqq p \leqq \infty$ and a nonnegative integer $k, W^{k, p}$ denotes the Banach space of all functions in $L^{p}$ with derivatives up to $k$ th order in $L^{p}$. Let the norm in $W^{k, p}$ be defined as follows:

$$
\begin{aligned}
& \|v\|_{W^{k, p}}=\left(\sum_{|\alpha| \leqq k}\left\|\left(\frac{\partial}{\partial x}\right)^{\alpha} v\right\|_{L^{p}}\right)^{1 / p}, \quad 1 \leqq p<\infty, \\
& \|v\|_{W^{k, \infty}}=\max _{|\alpha| \leqq k}\left\|\left(\frac{\partial}{\partial x}\right)^{\alpha} v\right\|_{L^{\infty}}, \quad p=\infty .
\end{aligned}
$$

For $v \in \mathscr{S}^{\prime}\left(\mathbf{R}^{3}\right), \hat{v}$ denotes the Fourier transform of $v$. For a function $v$ on $\mathbf{R}^{3}$, we denote the support of $v$ by supp $v$. For $z \in \mathbf{C}$, we denote the complex conjugate of $z$ by $\bar{z}$. Let $\omega=\sqrt{-\Delta}$ and $U(t)=e^{\frac{i}{2} \Delta t}$. Let $\theta(t)$ be a real-valued function in $C^{\infty}([0, \infty))$ such that $\theta(t)=1$ for $t \geqq 2$ and $\theta(t)=0$ for $0 \leqq t \leqq 1$.

Now we state the main results in this paper.

Theorem 1.1. Let $1>\delta>0$. Assume that $A_{+0}=\left(A_{+0}^{1}, A_{+0}^{2}, A_{+0}^{3}\right) \in \dot{H}^{5} \cap \dot{H}^{1} \cap W^{5,1}$, $A_{+1}=\left(A_{+1}^{1}, A_{+1}^{2}, A_{+1}^{3}\right) \in H^{4} \cap W^{4,1}$ and $\operatorname{div} A_{+0}=\operatorname{div} A_{+1}=0$. We put $A_{+}(t)=$ $\left(A_{+}^{1}(t), A_{+}^{2}(t), A_{+}^{3}(t)\right)=\cos \omega t A_{+0}+\omega^{-1} \sin \omega t A_{+1}$. Assume that $\psi_{+0} \in H^{6,7}$ and $\operatorname{supp} \hat{\psi}_{+0}(\xi) \subset\{\xi ;|\xi| \geqq 1+\delta\} \cup\{\xi ;|\xi| \leqq 1-\delta\}$. We put $\psi_{+}(t)=U(t) \psi_{+0}$. Then, there exists an $\eta>0$ such that if

$$
\left\|A_{+0}\right\|_{\dot{H}^{5} \cap \dot{H}^{1}}+\left\|A_{+0}\right\|_{W^{5,1}}+\left\|A_{+1}\right\|_{H^{4}}+\left\|A_{+1}\right\|_{W^{4,1}}+\left\|\psi_{+0}\right\|_{H^{6,7}} \leqq \eta,
$$

(1.1)-(1.3) have the unique solutions $(A(t), \psi(t))$ satisfying

$$
\begin{gathered}
A(t) \in C\left([0, \infty) ; \dot{H}^{3} \cap \dot{H}^{1}\right) \\
\frac{\partial}{\partial t} A(t) \in \bigcap_{k=0}^{2} C^{k}\left([0, \infty) ; H^{2-k}\right) \\
\psi(t) \in \bigcap_{k=0}^{1} C^{k}\left([0, \infty) ; H^{3-2 k}\right), \\
(1+t)^{1 / 2}\left\{\left\|A(t)-A_{+}(t)-R(t)\right\|_{\dot{H}^{2} \cap \dot{H}^{1}}+\sum_{j=1}^{2}\left\|\left(\frac{\partial}{\partial t}\right)^{j}\left(A(t)-A_{+}(t)-R(t)\right)\right\|_{H^{2-j}}\right\} \\
+\left\{\left\|A(t)-A_{+}(t)-R(t)\right\|_{\dot{H}^{3}}+\sum_{j=1}^{3}\left\|\left(\frac{\partial}{\partial t}\right)^{j}\left(A(t)-A_{+}(t)-R(t)\right)\right\|_{H^{3-j}}\right\}
\end{gathered}
$$




$$
\begin{aligned}
+\sum_{j=0}^{1} & \left\|\left(\frac{\partial}{\partial t}\right)^{j}\left\{\psi(t)-e^{i \varphi} \psi_{+}(t)\right\}\right\|_{H^{3-2 \jmath}}=O\left(t^{-1}(\log t)^{2}\right) \quad(t \rightarrow+\infty), \\
& \left(\int_{t}^{\infty}\left\|\psi(s)-e^{i \varphi} \psi_{+}(s)\right\|_{W^{2,4}}^{8 / 3} d s\right)^{3 / 8}=O\left(t^{-1}(\log t)^{2}\right) \quad(t \rightarrow+\infty),
\end{aligned}
$$

where $\eta$ depends only on $\delta, R(t)=\left(R^{1}(t), R^{2}(t), R^{3}(t)\right)$ is the solution of the following linear problem:

$$
\begin{aligned}
& \frac{\partial^{2}}{\partial t^{2}} R-\Delta R=\left[2|t|^{-3} \frac{x}{t}\left|\hat{\psi}_{+0}\left(\frac{x}{t}\right)\right|^{2}\right. \\
& \left.-i|t|^{-3}\left\{\overline{\hat{\psi}}_{+0}\left(\frac{x}{t}\right) \frac{1}{t}\left(\nabla \hat{\psi}_{+0}\right)\left(\frac{x}{t}\right)-\hat{\psi}_{+0}\left(\frac{x}{t}\right) \frac{1}{t}\left(\nabla \overline{\hat{\psi}}_{+0}\right)\left(\frac{x}{t}\right)\right\}\right] \theta(t)^{2} \\
& +\frac{1}{4 \pi} \nabla\left[\frac { 1 } { | x | } * \nabla \cdot \left\{2|t|^{-3} \frac{x}{t}\left|\hat{\psi}_{+o}\left(\frac{x}{t}\right)\right|^{2}\right.\right. \\
& -i|t|^{-3}\left(\overline{\hat{\psi}}_{+0}\left(\frac{x}{t}\right) \frac{1}{t}\left(\nabla \hat{\psi}_{+0}\right)\left(\frac{x}{t}\right)\right. \\
& \left.\left.\left.-\hat{\psi}_{+0}\left(\frac{x}{t}\right) \frac{1}{t}\left(\nabla \overline{\hat{\psi}}_{+0}\right)\left(\frac{x}{t}\right)\right)\right\}\right] \theta(t)^{2}, \quad t>0, \\
& \left\|\frac{\partial}{\partial t} R(t)\right\|_{L^{2}}^{2}+\sum_{j=1}^{3}\left\|\nabla R^{j}(t)\right\|_{L^{2}}^{2} \rightarrow 0 \quad(t \rightarrow+\infty),
\end{aligned}
$$

and

$$
\varphi(t, x)=-\frac{1}{8 \pi} \log t \int_{\mathbf{R}^{3}} \frac{1}{|y|}\left|\hat{\psi}_{+0}\left(\frac{x}{t}-y\right)\right|^{2} d y+\sum_{j=1}^{3} \int_{0}^{t} R^{j}\left(s, \frac{s}{t} x\right) d s \frac{x_{j}}{t} .
$$

Remark 1.1. (i) We note that for $k>l>0, \dot{H}^{k} \nsubseteq \dot{H}^{l} \nsubseteq L^{2}$. But, if $v \in \dot{H}^{k} \cap \dot{H}^{1}$ for a positive integer $k$, then $\left(\frac{\partial}{\partial x}\right)^{\alpha} v \in L^{2}$ for any multi-index $\alpha$ with $1 \leqq|\alpha| \leqq k$. In addition, for the three dimensional case, $\dot{H}^{1} \subset L^{6}$. For the homogeneous Sobolev space $\dot{H}^{m}\left(\mathbf{R}^{n}\right)$, see, e.g., $[2, \S 6.3]$. But note that the definition of $\dot{H}^{m}\left(\mathbf{R}^{n}\right)$ in the present paper is slightly different from the one in $[2, \S 6.3]$, where $\dot{H}^{m}\left(\mathbf{R}^{n}\right)$ is defined as the set of all tempered distributions $v$ such that $(-\Delta)^{m / 2} v \in L^{2}\left(\mathbf{R}^{n}\right)$. If we take $v \in \dot{H}^{m}\left(\mathbf{R}^{n}\right)$ modulo polynomials of degree at most $[m-n / 2]$ in the definition of $[2, \S 6.3]$, then the definition in $[2, \S 6.3]$ is identical to that in the present paper (see $[2$, problem 12 in $\$ 6.8])$. Here $[m-n / 2]$ is the largest integer that is not larger than $m-n / 2$ and if $[m-n / 2]$ is negative, we take zero as a polynomial of order $[m-n / 2]$.

(ii) There exists the unique solution $R(t)$ of (1.10)-(1.11) under the assumptions of Theorem 1.1 (see Lemma 2.4 in $\S 2$ ). We note that $R(t)$ does not necessarily belong to $L^{2}$ for $t \geqq 0$ and

$$
\|R(t)\|_{L^{x}}=O\left(t^{-1}\right) \quad(t \rightarrow+\infty) .
$$


(iii) $A_{+}(t)$ and $\psi_{+}(t)$ are the free solutions of the Maxwell equations and the Schrödinger equation with the initial conditions $A_{+}(0)=A_{+0}, \frac{\partial}{\partial t} A_{+}(0)=A_{+1}$ and $\psi_{+}(0)=\psi_{+0}$, respectively. Furthermore,

$$
\operatorname{div} A_{+}(t)=\frac{\partial}{\partial t} \operatorname{div} A_{+}(t)=0, \quad t \geqq 0
$$

(iv) Equation (1.8) shows that the phase function $\varphi(t, x)$ defined in (1.12) indicates the phase shift of $\psi(t, x)$ from the free dynamics. The first term at the right-hand side of (1.12) corresponds to the long range effect of the Coulomb term, that is, the last term at the left-hand side of (1.2). This part of (1.12) is the same as the phase function given in [13] for the decoupled nonlinear Schrödinger equation. The second term at the right-hand side of (1.12) corresponds to the long range effect resulting from the coupling of the Maxwell equations and the Schrödinger equation.

(v) The assumption on the support of $\hat{\psi}_{+0}$ ensures that the product of $A_{+}(t)$ and $\nabla \psi_{+}(t)$ decays fast enough as $t \rightarrow+\infty$. This fact is based on the difference between the propagation properties of the Maxwell wave and the Schrödinger wave (see Corollary 2.5(ii) in Sect. 2). The influence by the product of $A_{+}(t)$ and $\nabla \psi_{+}(t)$ is most difficult to control, although it does not seem to be so bad at first sight.

(vi) We consider the Maxwell--Schrödinger equations under the Coulomb gauge condition instead of the Lorentz gauge condition, because the former is more usual than the latter in the nonrelativistic case from a physical point of view. Our proof of Theorem 1.1 is also applicable to the case of the Lorentz gauge condition.

(vii) For the case of $t \rightarrow-\infty$, we can obtain the same result as Theorem 1.1 without change of the proof.

The following corollary is an immediate consequence of Theorem 1.1.

Corollary 1.2. (i) Let $\delta>0$. By $D_{+}$we denote the set of all scattered states $\left(A_{+0}, A_{+1}, \psi_{+0}\right)$ such that $\operatorname{div} A_{+0}=\operatorname{div} A_{+1}=0, \operatorname{supp} \hat{\psi}_{+0} \subset\{\xi ;|\xi| \geqq 1+\delta\} \cup$ $\{\xi ;|\xi| \leqq 1-\delta\}$ and (1.4) holds. Then, for (1.1)-(1.3) the modified wave operator $W_{+}$: $\left(A_{+0}, A_{+1}, \psi_{+0}\right) \mapsto\left(A(0), \frac{\partial}{\partial t} A(0), \psi(0)\right)$ is well defined on $D_{+}$.

(ii) Let $R\left(W_{+}\right)$be the range of the modified wave operator $W_{+}$given by part (i). Then, for any $\left(A_{0}, A_{1}, \psi_{0}\right) \in R\left(W_{+}\right)$there exist the global solutions $(A(t), \psi(t))$ of (1.1)-(1.3) with $\left(A(0), \frac{\partial}{\partial t} A(0), \psi(0)\right)=\left(A_{0}, A_{1}, \psi_{0}\right)$ satisfying (1.5)-(1.7). Furthermore, these solutions satisfy (1.8) and (1.9) with $\left(A_{+0}, A_{+1}, \psi_{+0}\right)=W_{+}^{-1}\left(A_{0}, A_{1}, \psi_{0}\right)$.

Remark 1.2. (i) Noting Remark 1.2(vii), we can also construct the modified wave operator $W_{-}$, which is a mapping from the scattered states as $t=-\infty$ to the interacting states at $t=0$.

(ii) Corollary 1.2(ii) gives the global existence result for the initial value problem of (1.1)-(1.3). However, it is not clear what initial data belong to $R\left(W_{+}\right)$.

The proof of Theorem 1.1 is roughly described as follows. Since the last term at the right-hand side of (1.1) is difficult to treat as it is, we rewrite (1.1) by using (1.2). 
We substitute (1.2) into (1.1) to obtain

$$
\begin{aligned}
\frac{\partial^{2}}{\partial t^{2}} A-\Delta A= & -i(\bar{\psi} \nabla \psi-\psi \nabla \bar{\psi})-2 A|\psi|^{2} \\
& +\frac{1}{4 \pi} \nabla\left[\frac{1}{|x|} * \nabla \cdot\left\{-i(\bar{\psi} \nabla \psi-\psi \nabla \bar{\psi})-2 A|\psi|^{2}\right\}\right], \quad t>0, x \in \mathbf{R}^{3} .
\end{aligned}
$$

We also rewrite (1.2) as follows:

$$
\begin{aligned}
2 i \frac{\partial}{\partial t} \psi+\Delta \psi= & i(\operatorname{div} A) \psi+2 i A \cdot \nabla \psi \\
& +|A|^{2} \psi+\frac{1}{4 \pi}\left(\frac{1}{|x|} *|\psi|^{2}\right) \psi, \quad t>0, \quad x \in \mathbf{R}^{3} .
\end{aligned}
$$

Equations (1.14)-(1.15) are equivalent to (1.1)-(1.2), if the solutions $(A(t), \psi(t))$ are smooth. It is well known that if the $L^{2}$ norm of the perturbed term is integrable in time over $[0, \infty)$, the solution of an evolution equation such as the MaxwellSchrödinger equations behaves like a free solution as $t \rightarrow \infty$. Therefore, it is conjectured that the long range effect arises from the second term and the last term at the right-hand side of (1.15). The long range effect of the latter can be controlled in the same way as the case of the decoupled nonlinear Schrödinger equation (see Ozawa [13]). The long range effect of the former comes from the coupling of the Maxwell equations and the Schrödinger equation. Accordingly, our main task in the proof of Theorem 1.1 is to evaluate the long range effect of the second term $2 i A \cdot \nabla \psi$ at the right-hand side of (1.15). For that purpose, we first assume that the support of $\hat{\psi}_{+0}$ does not include the unit sphere centered at the origin. This assumption ensures that the product of $A_{+}(t)$ and $\nabla \psi_{+}(t)$ decays faster as $t \rightarrow \infty$ than $A_{+}(t)$ alone or $\nabla \psi_{+}(t)$ alone and so the influence by the product of $A_{+}(t)$ and $\nabla \psi_{+}(t)$ decays fast enough as $t \rightarrow \infty$. This fact results from the difference between the propagation properties of the Maxwell wave and the Schrödinger wave (see Corollary 2.5 (ii) in Sect. 2). After the influence by the product of $A_{+}(t)$ and $\nabla \psi_{+}(t)$ has become negligible, the long range effect of $2 i A \cdot \nabla \psi$ still remains. Therefore, we need to introduce a long range correction in the definition of the wave operator. The solution $A(t)$ of the Maxwell part can be divided into three parts, that is, $A(t)=A_{+}(t)+R(t)+v(t)$. Here $A_{+}(t)$ is a free solution which $A(t)$ approaches asymptotically, $R(t)$ is a solution of (1.10)-(1.11) which has a long range effect on the solution of the Schrödinger part and $v(t)$ is a remainder which decays faster as $t \rightarrow \infty$ than $A_{+}(t)$ and $R(t)$. If we put

$$
m(t, x)=(i t)^{-3 / 2} e^{\frac{i|x|^{2}}{2 t}} \hat{\psi}_{+0}\left(\frac{x}{t}\right) \theta(t),
$$

we can take $m(t, x)$ as an asymptotic profile of $\psi_{+}(t)$ (see Lemma 2.1(iii) in Sect. 2). We put

$$
\begin{aligned}
& A(t)=A_{+}(t)+R(t)+v(t), \\
& \psi(t)=e^{i \varphi} m(t)+u(t),
\end{aligned}
$$


where $\varphi(t, x)$ is defined in (1.12). If we rewrite (1.14)-(1.15) as the equations of $v(t)$ and $u(t)$, the terms of slow time decay vanish and the resulting equations of $v(t)$ and $u(t)$ with the final states given at $t=\infty$ become solvable in proper function spaces under the assumptions of Theorem 1.1.

Our plan in this paper is the following. In Sect. 2 we summarize several lemmas and one proposition needed for the proof of Theorem 1.1. In Sect. 3 we state the proof of Theorem 1.1.

We conclude this section with several notations given. For two three-dimensional complex vectors $a, b \in \mathbf{C}^{3}$, we put $a \cdot b=a_{1} b_{1}+a_{2} b_{2}+a_{3} b_{3}$. We abbreviate $\partial / \partial x_{j}, j=1,2,3$ and $\partial / \partial t$ to $\partial_{x}, j=1,2,3$ and $\partial_{t}$, respectively. For a multi-index $\alpha=\left(\alpha_{1}, \alpha_{2}, \alpha_{3}\right)$, we put $\partial_{x}^{\alpha}=\partial_{x_{1}}^{\alpha_{1}} \partial_{x_{2}}^{\alpha_{2}} \partial_{x_{3}}^{\alpha_{3}}$ and $x^{\alpha}=x_{1}^{\alpha_{1}} x_{2}^{\alpha_{2}} x_{3}^{\alpha_{3}}$. We denote the scalar product in $L^{2}$ by $(\cdot, \cdot)$. For $s \in \mathbf{R}$, let $[s]$ be the largest integer that is not larger than s. Let $\rho$ be a nonnegative function in $C_{0}^{\infty}\left(\mathbf{R}^{3}\right)$ such that $\|\rho\|_{L^{1}}=1$. For $\varepsilon>0$, we put $\rho_{\varepsilon}(x)=\varepsilon^{-3} \rho\left(\frac{x}{\varepsilon}\right)$. In the course of calculations below, various constants are simply denoted by $C$.

\section{Preliminary Results}

In this section we give several lemmas and one proposition needed for the proof of Theorem 1.1.

We start with the estimates of the evolution operator for the free Schrödinger equation.

Lemma 2.1. Let $n \geqq 1$.

Then,

(i) Let $p$ and $q$ be two positive constants such that $\frac{1}{p}+\frac{1}{q}=1$ and $2 \leqq p \leqq+\infty$.

$$
\|U(t) v\|_{L^{p}\left(\mathbf{R}^{n}\right)} \leqq(2 \pi|t|)^{-\left(\frac{n}{2}-\frac{n}{p}\right)}\|v\|_{L^{q}\left(\mathbf{R}^{n}\right)}, \quad v \in L^{q}\left(\mathbf{R}^{n}\right), t \neq 0 .
$$

(ii) Let $q$ and $r$ be two positive constants such that $2 \leqq q<+\infty$ for $n=1,2,2 \leqq q<\frac{2 n}{n-2}$ for $n \geqq 3$ and $\left(\frac{n}{2}-\frac{n}{q}\right) r=2$. Then,

$$
\left(\int_{t}^{\infty}\left\|\int_{\tau}^{\infty} U(\tau-s) f(s) d s\right\|_{L^{q}\left(\mathbf{R}^{n}\right)}^{r} d \tau\right)^{1 / r} \leqq K_{1} \int_{t}^{\infty}\|f(s)\|_{L^{2}\left(\mathbf{R}^{n}\right)} d s, \quad t \geqq 0,
$$

where $K_{1}$ is a positive constant depending only on $n$ and $q$.

(iii) Let $k$ be a nonnegative integer. Suppose that for $j+|\alpha| \leqq k$, $(1+|x|)^{j+2} \partial_{x}^{\alpha} \psi_{+0} \in L^{2}\left(\mathbf{R}^{n}\right)$ and $(1+|x|)^{j+2} \partial_{x}^{\alpha} \psi_{+0} \in L^{1}\left(\mathbf{R}^{n}\right)$. We put $m(t, x)=$ $(i t)^{-n / 2} e^{\frac{i|x|^{2}}{2 t}} \hat{\psi}_{+0}\left(\frac{x}{t}\right) \theta(t)$. Then, for some $K_{2}>0$,

$$
\begin{aligned}
& \sum_{2 j+|x|+|\beta| \leqq k}\left\|\left(\frac{x}{t}\right)^{\beta} \partial_{x}^{\alpha} \partial_{t}^{j}\left\{U(t) \psi_{+0}-m(t)\right\}\right\|_{L^{2}\left(\mathbf{R}^{n}\right)} \\
& \leqq K_{2} t^{-1} \sum_{j+|\alpha| \leqq k}\left\|(1+|x|)^{j+2} \partial_{x}^{\alpha} \psi_{+0}\right\|_{L^{2}\left(\mathbf{R}^{n}\right), \quad t \geqq 1}
\end{aligned}
$$




$$
\begin{aligned}
& \sum_{2 j+|x|+|\beta| \leqq k}\left\|\left(\frac{x}{t}\right)^{\beta} \partial_{x}^{\alpha} \partial_{t}^{j}\left\{U(t) \psi_{+0}-m(t)\right\}\right\|_{L^{\infty}\left(\mathbf{R}^{n}\right)} \\
& \leqq K_{2} t^{-n / 2-1} \sum_{j+|\alpha| \leqq k}\left\|(1+|x|)^{j+2} \partial_{x}^{\alpha} \psi+0\right\|_{L^{1}\left(\mathbf{R}^{n}\right)}, \quad t \geqq 1,
\end{aligned}
$$

where $K_{2}$ depends only on $k$ and $n$.

Remark 2.1. Lemma 2.1(ii) implies that $m(t)$ is asymptotically equivalent to $U(t) \psi_{+0}$ as $t \rightarrow \infty$.

For the proofs of Lemma 2.1(i) and (ii), see, e.g., [17] and [6]. For the proof of Lemma 2.1(iii), see [14, Lemma 2.1(ii)]. Lemma 2.1(ii) in [14] treats only the case $|\beta|=0$, but the proof for $|\beta| \neq 0$ is the same as that for $|\beta|=0$.

The following lemma is concerned with the decay property of solution of the free wave equation.

Lemma 2.2. (i) Let $n \geqq 1$ and let $L$ be a nonnegative integer. Then,

$$
\begin{aligned}
& \sum_{j+|x| \leqq L}\left\|\partial_{x}^{x} \partial_{t}^{j} \cos \omega t u\right\|_{L^{\infty}\left(\mathbf{R}^{n}\right)} \\
& \quad \leqq K_{3}(1+t)^{-(n-1) / 2}\left(\|u\|_{H^{L+[n / 2]+1}\left(\mathbf{R}^{n}\right)}+\|u\|_{W^{L+[n: 2]+1,1}\left(\mathbf{R}^{n}\right)}\right), \quad t>0 \\
& \sum_{J+|x| \leqq L}\left\|\partial_{x}^{\alpha} \partial_{t}^{j} \omega^{-1} \sin \omega t u\right\|_{L^{\infty}\left(\mathbf{R}^{n}\right)} \\
& \quad \leqq K_{3}(1+t)^{-(n-1) / 2}\left(\|u\|_{H^{L+[n / 2]}\left(\mathbf{R}^{n}\right)}+\|u\|_{\left.W^{L+[n / 2], 1}\left(\mathbf{R}^{n}\right)\right), \quad t>0}\right.
\end{aligned}
$$

where $K_{3}$ depends only on $L$ and $n$.

(ii) Let $n$ be odd spatial dimensions larger than one and let $L$ be a nonnegative integer. For any $\delta$ with $1>\delta>0$, there exists a $K_{5}>0$ such that

$$
\begin{gathered}
\sum_{j+|\alpha| \leqq L}\left\|\partial_{x}^{\alpha} \partial_{t}^{j} \cos \omega t u\right\|_{L^{x}\left(M_{\delta}(t)\right)} \leqq K_{4}(1+t)^{-2}\|u\|_{H^{L+[n / 2]+1,2}}, \quad t>0 \\
\sum_{j+|\alpha| \leqq L}\left\|\partial_{x}^{\alpha} \partial_{t}^{j} \omega^{-1} \sin \omega t u\right\|_{L^{\infty}\left(M_{\delta}(t)\right)} \leqq K_{4}(1+t)^{-2}\|u\|_{H^{L+[n / 2]+1,2}}, \quad t>0
\end{gathered}
$$

where $M_{\delta}(t)=\left\{\xi \in \mathbf{R}^{n} ;|\xi| \geqq(1+\delta) t\right\} \cup\left\{\xi \in \mathbf{R}^{n} ;|\xi| \leqq(1-\delta) t\right\}$ and $K_{4}$ depends only on $L, \delta$ and $n$.

Proof. For the proof of part (i), see $[9, \S 1]$.

Part (ii) follows from the Huygens principle for the wave equation in odd spatial dimensions larger than one. We first choose two functions $\phi_{1}, \phi_{2} \in C^{\infty}(\mathbf{R})$ such that $\phi_{j}(s)=\phi_{j}(-s), j=1,2, \phi_{1}(s)=1$ for $|s| \leqq 1, \phi_{1}(s)=0$ for $|s| \geqq\left(1-\frac{\delta}{2}\right) /(1-\delta)$, $\phi_{s}(s)=1$ for $|s| \geqq 1$ and $\phi_{2}(s)=0$ for $|s| \leqq\left(1+\frac{\delta}{2}\right) /(1+\delta)$. We put

$$
\phi_{\delta}(t, x)=\phi_{1}\left(\frac{|x|}{(1-\delta) t}\right)+\phi_{2}\left(\frac{|x|}{(1+\delta) t}\right) \text {. }
$$


We note that for any nonnegative integer $k$,

$$
\sum_{j+|\alpha| \leqq k}\left|\partial_{x}^{\alpha} \partial_{t}^{j} \phi_{\delta}(t, x)\right| \leqq C_{k}, \quad(t, x) \in[0, \infty) \times \mathbf{R}^{n} .
$$

Since the propagation speed of wave is one, we have by the Sobolev imbedding theorem and the Huygens principle

$$
\begin{aligned}
& \sum_{J+|\alpha| \leqq L}\left\|\partial_{x}^{\alpha} \partial_{t}^{j} \cos \omega t u\right\|_{L^{\infty}\left(M_{\delta}(t)\right)} \\
& \leqq \sum_{J+|\alpha| \leqq L}\left\|\partial_{x}^{\alpha} \partial_{t}^{j}\left(\phi_{\delta} \cos \omega t u\right)\right\|_{L^{\infty}\left(\mathbf{R}^{n}\right)} \\
& \leqq C \quad \sum_{J+|\alpha| \leqq L+[n / 2]+1}\left\|\partial_{x}^{\alpha} \partial_{t}^{j}\left(\phi_{\delta} \cos \omega t u\right)\right\|_{L^{2}\left(\mathbf{R}^{n}\right)} \\
& \leqq C \sum_{J+|\alpha| \leqq L+[n / 2]+1}\left\|\partial_{x}^{\alpha} u\right\|_{L^{2}\left(|x|>\frac{\partial}{2} t\right)} \\
& \leqq C \quad \sum_{J+|x| \leqq L+[n / 2]+1}\left(1+\frac{\delta}{2} t\right)^{-2}\left\|(1+|x|)^{2} \partial_{x}^{\alpha} u\right\|_{L^{2}\left(|x|>\frac{\delta}{2} t\right)} \\
& \leqq C(1+t)^{-2}\|u\|_{H^{L+[n / 2]+1,2}\left(\mathbf{R}^{n}\right)}, \quad t \geqq 1 .
\end{aligned}
$$

By the Sobolev imbedding theorem we also have

$$
\begin{aligned}
\sum_{j+|x| \leqq L}\left\|\partial_{x}^{\alpha} \partial_{t}^{j} u\right\|_{L^{\infty}\left(\mathbf{R}^{n}\right)} & \leqq C \sum_{j+|x| \leqq L+[n / 2]+1}\left\|\partial_{x}^{\alpha} \partial_{t}^{j} \cos \omega t u\right\|_{L^{2}\left(\mathbf{R}^{n}\right)} \\
& \leqq C \sum_{J+|x| \leqq L+[n / 2]+1}\left\|\partial_{x}^{\alpha} u\right\|_{L^{2}\left(\mathbf{R}^{n}\right)}, \quad t \geqq 0 .
\end{aligned}
$$

Equations (2.9) and (2.10) show (2.7). The proof of (2.8) is the same as above.

Remark 2.1. (i) The analogous result to Lemma 2.2(ii) also holds in even space dimensions (see [15, Theorem XI. 18(a)]), although the Huygens principle does not strictly hold in even space dimensions.

(ii) Lemmas 2.2(i) and (ii) imply that the solution of the wave equation decays faster inside and outside the light cone as $t \rightarrow \infty$ than on the light cone.

We next consider the following linear wave equation:

$$
\begin{gathered}
\partial_{t}^{2} w-\Delta w=h(t, x), \quad t>0, \quad x \in \mathbf{R}^{3}, \\
\|\nabla w(t)\|_{L^{2}}^{2}+\left\|\partial_{t} w(t)\right\|_{L^{2}}^{2} \rightarrow 0 \quad(t \rightarrow \infty) .
\end{gathered}
$$

We have the following proposition concerning the solvability of (2.11)-(2.12).

Proposition 2.3. Let $L$ be an arbitrary integer with $L \geqq 2$. We assume that

$$
h(t) \in \bigcap_{j=0}^{L-1} C^{j}\left([0, \infty) ; H^{L-j}\right)
$$

and for some $C_{0}>0$,

$$
\sum_{k=0}^{L-1} \sum_{t+|\alpha|=k}(1+t)^{3 / 2+k}\left\|\partial_{x}^{\alpha} \partial_{t}^{j} h(t)\right\|_{L^{2}} \leqq C_{0}, \quad t>0 .
$$


Then, there exists a unique solution $w(t)$ of (2.11)-(2.12) satisfying

$$
\begin{gathered}
w(t) \in C\left([0, \infty) ; \dot{H}^{L} \cap \dot{H}^{1}\right) \\
\partial_{t} w(t) \in \bigcap_{j=0}^{L-1} C^{j}\left([0, \infty) ; H^{L-1-j}\right), \\
\sum_{J+|x|=k}\left\|\partial_{x}^{\alpha} \partial_{t}^{j} w(t)\right\|_{L^{2}} \leqq K_{5}(1+t)^{1 / 2-k} \sup _{t \geqq 0} \sum_{j+|\alpha|=k-1}(1+t)^{k+1 / 2}\left\|\partial_{x}^{\alpha} \partial_{t}^{j} h(t)\right\|_{L^{2}}, \\
t>0, \quad k=1, \ldots, L, \\
\sum_{j+|\alpha|=k}\left\|\partial_{x}^{\alpha} \partial_{t}^{j} w(t)\right\|_{L^{\infty}} \leqq K_{5}(1+t)^{-1-k} \\
\times \sup _{t \geqq 0} \sum_{l=k}^{k+1} \sum_{j+|x|=l}(1+t)^{3 / 2+l}\left\|\partial_{x}^{\alpha} \partial_{t}^{j} h(t)\right\|_{L^{2}}, \quad t>0, \quad k=0, \ldots, L-2,
\end{gathered}
$$

where $K_{5}$ depends only on $L$.

Proof. We first consider the following regularized problem:

$$
\begin{gathered}
\partial_{t}^{2} w_{\varepsilon \lambda}-\Delta w_{\varepsilon \lambda}=(1+\lambda t)^{-L-1} \rho_{\varepsilon} * h(t), \quad t>0, \quad x \in \mathbf{R}^{3}, \\
\left\|\nabla w_{\varepsilon \lambda}(t)\right\|_{L^{2}}^{2}+\left\|\partial_{t} w_{\varepsilon \lambda}(t)\right\|_{L^{2}}^{2} \rightarrow 0 \quad(t \rightarrow+\infty),
\end{gathered}
$$

where $0<\lambda<1$ and $0<\varepsilon<1$. The solution $w_{\varepsilon \lambda}(t)$ of $(2.17)-(2.18)$ is given by

$$
w_{\varepsilon \lambda}(t)=-\int_{t}^{\infty}(1+\lambda s)^{-L-1}\left(\omega^{-1} \sin \omega(t-s)\right) \rho_{\varepsilon} * h(s) d s, \quad t \geqq 0 .
$$

Formula (2.19) and a direct calculation yield

$$
\begin{gathered}
w_{\varepsilon \lambda}(t) \in \bigcap_{j=1}^{\infty} C^{L+1}\left([0, \infty) ; H^{j}\right) \\
\sup _{t \geqq 0}\left[(1+t)^{L+1 / 2}\left\|w_{\varepsilon \lambda}(t)\right\|_{L^{2}}+\sum_{1 \leqq j+|\alpha| \leqq L+1}(1+t)^{L+3 / 2}\left\|\partial_{x}^{\alpha} \partial_{t}^{j} w_{\varepsilon \hat{\lambda}}(t)\right\|_{L^{2}}\right]<\infty
\end{gathered}
$$

for $0<\lambda<1$ and $0<\varepsilon<1$.

We next derive the a priori estimates independent of $\lambda$ and $\varepsilon$ for $w_{\varepsilon \lambda}$. We take the scalar product in $L^{2}$ between (2.17) and $\partial_{t} w_{\varepsilon \lambda}(t)$ to obtain

$\frac{1}{2} \frac{d}{d t}\left\{\left\|\partial_{t} w_{\varepsilon \lambda}(t)\right\|_{L^{2}}^{2}+\left\|\nabla w_{\varepsilon \lambda}\right\|_{L^{2}}^{2}\right\}=(1+\lambda t)^{-L-1}\left(\rho_{\varepsilon} * h(t), \partial_{t} w_{\varepsilon \lambda}(t)\right), \quad t>0$.

Integrating $(2.22)$ over $[t, \infty)$, we have by Young's inequality

$$
\begin{aligned}
\left\|\partial_{t} w_{\varepsilon \lambda}(t)\right\|_{L^{2}}^{2}+\left\|\nabla w_{\varepsilon \lambda}(t)\right\|_{L^{2}}^{2} & \leqq 2 \int_{t}^{\infty}(1+\lambda s)^{-L-1}\|h(s)\|_{L^{2}}\left\|\partial_{s} w_{\varepsilon \lambda}(s)\right\|_{L^{2}} d s \\
& \leqq 2 \int_{t}^{\infty}(1+\lambda s)^{-L-1}(1+s)^{-2} d s
\end{aligned}
$$




$$
\begin{aligned}
& \times\left\{\sup _{s \geqq t}(1+s)^{3 / 2}\|h(s)\|_{L^{2}}\right\} \\
& \times\left\{\sup _{s \geqq t}(1+s)^{1 / 2}\left\|\partial_{s} w_{\varepsilon \lambda}(s)\right\|_{L^{2}}\right\} \\
\leqq & C(1+t)^{-1}\left\{\sup _{s \geqq t}(1+s)^{3 / 2}\|h(s)\|\right\} \\
& \times\left\{\sup _{s \geqq t}(1+s)^{1 / 2}\left\|w_{\varepsilon \lambda}(s)\right\|_{L^{2}}\right\} \\
\leqq & C(1+t)^{-1}\left\{\begin{array}{l}
\left.\sup _{s \geqq t}(1+s)^{3 / 2}\|h(s)\|_{L^{2}}\right\}^{2} \\
+
\end{array}\right. \\
& \frac{1}{2}(1+t)^{-1}\left\{\sup _{s \geqq t}(1+s)^{1 / 2}\left\|\partial_{s} w_{\varepsilon \lambda}(s)\right\|_{L^{2}}\right\}^{2}, t \geqq 0,
\end{aligned}
$$

where $C$ does not depend on $\varepsilon$ and $\lambda$. Equation (2.23) gives us

$$
\sup _{t \geqq 0}(1+t)^{1 / 2}\left\{\left\|\partial_{t} w_{\varepsilon \lambda}(t)\right\|_{L^{2}}+\left\|\nabla w_{\varepsilon \lambda}(t)\right\|_{L^{2}}\right\} \leqq C \sup _{t \geqq 0}(1+t)^{3 / 2}\|h(t)\|_{L^{2}},
$$

where $C$ does not depend on $\varepsilon$ and $\lambda$. In the same way as above we obtain

$$
\begin{aligned}
& \sup _{t \geqq 0} \sum_{J+|\alpha|=k}(1+t)^{k-1 / 2}\left\|\partial_{x}^{\alpha} \partial_{t}^{j} w_{\varepsilon \lambda}(t)\right\|_{L^{2}} \\
& \quad \leqq C \sup _{t \geqq 0} \sum_{J+|x|=k-1}(1+t)^{k+1 / 2}\left\|\partial_{x}^{\alpha} \partial_{t}^{j} h(t)\right\|_{L^{2}}, \quad k=2, \ldots, L,
\end{aligned}
$$

where $C$ does not depend on $\varepsilon$ and $\lambda$. Therefore, (2.24), (2.25) and the standard compactness argument show the existence of a solution to (2.11)-(2.12) satisfying (2.13) and (2.15) with the continuity in $t$ of $w_{\varepsilon \lambda}(t)$ and its derivatives replaced by $L^{\infty}(0, \infty)$. The continuity in $t$ follows from the regularity theorem of linear hyperbolic equation. Equation (2.16) follows immediately from (2.15) and the Gagliardo-Nirenberg inequality (see, e.g., [4]).

We finally prove the uniqueness. We suppose that $w_{1}(t)$ and $w_{2}(t)$ are two solutions of (2.11) and (2.12) satisfying (2.13)-(2.15). Then we put $z=w_{1}-w_{2}$ to obtain

$$
\begin{gathered}
\partial_{t}^{2} z-\Delta z=0, \quad t>0, \quad x \in \mathbf{R}^{3}, \\
\left\|\partial_{t} z(t)\right\|_{L^{2}}^{2}+\|\nabla z(t)\|_{L^{2}}^{2} \rightarrow 0 \quad(t \rightarrow+\infty) .
\end{gathered}
$$

The conservation law of energy for (2.26) shows

$$
\left\|\partial_{t} z(t)\right\|_{L^{2}}^{2}+\|\nabla z(t)\|_{L^{2}}^{2}=\left\|\partial_{t} z(s)\right\|_{L^{2}}^{2}+\|\nabla z(s)\|_{L^{2}}^{2}, \quad 0 \leqq s, t<+\infty .
$$

Letting $s \rightarrow+\infty$ in (2.28), we have by (2.27)

$$
\left\|\partial_{t} z(t)\right\|_{L^{2}}^{2}+\|\nabla z(t)\|_{L^{2}}^{2}=0
$$

for any $t \geqq 0$, which implies that $w_{1}(t)=w_{2}(t)$ for $t \geqq 0$. The proof is complete. 
We next state the following lemma concerning the estimates of the phase function defined in (1.12).

Lemma 2.4. We assume $\psi_{+0} \in H^{6,7}$. Then, there exists the unique solution $R(t)$ of (1.10)-(1.11) such that

$$
\begin{gathered}
R(t) \in C\left([0, \infty) ; \dot{H}^{7} \cap \dot{H}^{1}\right), \\
\partial_{t} R(t) \in \bigcap_{k=0}^{6} C^{k}\left([0, \infty) ; H^{6-k}\right),
\end{gathered}
$$

$$
\begin{aligned}
& \sup _{t \geqq 0} \sum_{k=1}^{7}\left[(1+t)^{k-1 / 2} \sum_{J+|\alpha|=k}\left\|\partial_{x}^{\alpha} \partial_{t}^{j} R(t)\right\|_{L^{2}}\right] \\
& \quad+\sup _{t \geqq 0} \sum_{k=0}^{5}\left[(1+t)^{k+1} \sum_{j+|\alpha|=k}\left\|\partial_{t}^{\alpha} \partial_{t}^{j} R(t)\right\|_{L^{\infty}}\right] \leqq K_{6}\left\|\psi_{+0}\right\|_{H^{6,7}}^{2}
\end{aligned}
$$

for some $K_{6}>0$. Furthermore, the phase function $\varphi(t, x)$ defined in (1.12) satisfies

$$
\begin{aligned}
& \sup _{t \geqq 1} \sum_{k=0}^{5}\left[(1+t)^{k}(\log (1+t))^{-1} \sum_{j+|\alpha|=k}\left\|\left(1+\frac{|x|}{t}\right)^{-j-1} \partial_{x}^{\alpha} \partial_{t}^{j} \varphi(t)\right\|_{L^{\infty}}\right] \\
& \quad \leqq K_{7}\left\|\psi_{+0}\right\|_{H^{6,7}}^{2}
\end{aligned}
$$

for some $K_{7}>0$.

Proof. We note that for some $C>0$,

$$
\left\|\hat{\psi}_{+0}\right\|_{W^{5, x}} \leqq C\left\|\psi_{+0}\right\|_{H^{6,7}} .
$$

We put

$$
\begin{aligned}
H(t, x)= & h(t, x)+\frac{1}{4 \pi} \nabla\left(\frac{1}{|x|} * \nabla \cdot h\right)(t, x), \\
h(t, x)= & 2|t|^{-3} \frac{x}{t}\left|\hat{\psi}_{+0}\left(\frac{x}{t}\right)\right|^{2} \theta(t)^{2} \\
& -i|t|^{-3}\left\{\overline{\hat{\psi}}_{+0}\left(\frac{x}{t}\right) \frac{1}{t}\left(\nabla \hat{\psi}_{+0}\right)\left(\frac{x}{t}\right)-\hat{\psi}_{+0}\left(\frac{x}{t}\right) \frac{1}{t}\left(\nabla \overline{\hat{\psi}}_{+0}\left(\frac{x}{t}\right)\right\} \theta(t)^{2} .\right.
\end{aligned}
$$

We first verify that $H(t, x)$ satisfies the assumption in Proposition 2.4 with $L=7$. Let $k$ be any integer with $0 \leqq k \leqq 6$. Leibniz' rule shows that

$$
\sum_{J+|x|=k}\left|\partial_{x}^{\alpha} \partial_{t}^{j} h(t, x)\right|
$$

is bounded by a sum of terms

$$
\begin{aligned}
& C\left|\partial_{x}^{\alpha_{1}} \partial_{t}^{j_{1}}\left\{|t|^{-3 / 2} \frac{x}{t} \hat{\psi}_{+0}\left(\frac{x}{t}\right) \theta(t)^{2}\right\}\right|\left|\partial_{x}^{\alpha_{2}} \partial_{t}^{j_{2}}\left\{|t|^{-3 / 2} \overline{\hat{\psi}}_{+0}\left(\frac{x}{t}\right)\right\}\right|, \\
& C\left|\partial_{x}^{\alpha_{1}} \partial_{t}^{j_{1}}\left\{|t|^{-3 / 2} \overline{\hat{\psi}}_{+0}\left(\frac{x}{t}\right) \frac{\theta(t)^{2}}{t}\right\}\right|\left|\partial_{x}^{\alpha_{2}} \partial_{t}^{j_{2}}\left\{|t|^{-3 / 2}\left(\nabla \hat{\psi}_{+0}\right)\left(\frac{x}{t}\right)\right\}\right|,
\end{aligned}
$$


where $j_{1}+j_{2}=j$ and $\alpha_{1}+\alpha_{2}=\alpha$. Since either $j_{1}+\left|\alpha_{1}\right|$ or $j_{2}+\left|\alpha_{2}\right|$ is less than $[k / 2]+1$ and $\operatorname{supp} \theta(t) \subset\{t \geqq 1\}$, we have by $(2.33)$

$$
\begin{aligned}
\sum_{j+|\alpha|=k}\left\|\partial_{x}^{\alpha} \partial_{t}^{j} h(t)\right\|_{L^{2}} & \leqq C(1+t)^{-3 / 2-k}\left\|\hat{\psi}_{+0}\right\|_{W^{[k / 2]+2, \infty}}\left\|\hat{\psi}_{+0}\right\|_{H^{k+1, k}} \\
& \leqq C(1+t)^{-3 / 2-k}\left\|\psi_{+0}\right\|_{H^{6,7}}^{2}, \quad t \geqq 0 .
\end{aligned}
$$

On the other hand, since the Fourier transform of $\frac{1}{4 \pi|x|}$ is $|\xi|^{-2}$ and $\xi_{j} \xi_{k} /|\xi|^{2} \in L^{\infty}$, we have

$$
\left\|\frac{1}{4 \pi} \nabla\left(\frac{1}{|x|} * \nabla \cdot h\right)\right\|_{L^{2}} \leqq C\|h\|_{L^{2}} .
$$

Equations (2.34) and (2.35) show that $H(t, x)$ satisfies the assumption in Proposition 2.3 with $L=7$. Therefore, by Proposition 2.3 we obtain the first half of Lemma 2.4 .

We next prove (2.32). By the Gagliardo-Nirenberg inequality (see, e.g., [4]) and the Hardy-Littlewood-Sobolev inequality (see, e.g., [16]), we have

$$
\begin{aligned}
& \left\|\int_{\mathbf{R}^{3}} \frac{1}{|y|} v(x-y) d y\right\|_{L^{\infty}}=\left\|\int_{\mathbf{R}^{3}} \frac{1}{|x-y|} v(y) d y\right\|_{L^{\infty}} \\
& \quad \leqq C\left\|\Delta \int_{\mathbf{R}^{3}} \frac{1}{|x-y|} v(y) d y\right\|_{L^{2}}^{1 / 2}\left\|\nabla \int_{\mathbf{R}^{3}} \frac{1}{|x-y|} v(y) d y\right\|_{L^{2}}^{1 / 2} \\
& \leqq C\|v\|_{L^{2}}^{1 / 2}\|v\|_{L^{6 / 5}}^{1 / 2}
\end{aligned}
$$

Let $k$ be any integer with $0 \leqq k \leqq 5$ and let $j+|\alpha|=k$. Since $\partial_{t} \hat{\psi}_{+0}\left(\frac{x}{t}-y\right)=$ $-\frac{1}{t} \frac{x}{t} \cdot\left(\nabla \hat{\psi}_{+0}\right)\left(\frac{x}{t}-y\right)$, Leibniz' rule shows that

$$
\begin{aligned}
& \left.\left|\partial_{x}^{\alpha} \partial_{t}^{j}\right| \hat{\psi}_{+0}\left(\frac{x}{t}-y\right)\right|^{2} \mid \\
& \quad \leqq C t^{-k} \sum_{\left|\alpha_{1}\right|+\left|\alpha_{2}\right|=k}\left(1+\frac{|x|}{t}\right)^{j}\left|\left(\partial_{x}^{\alpha_{1}} \hat{\psi}_{+0}\right)\left(\frac{x}{t}-y\right)\right|\left|\left(\partial_{x}^{\alpha_{2}} \overline{\hat{\psi}}_{+0}\right)\left(\frac{x}{t}-y\right)\right|, \quad t \geqq 1 .
\end{aligned}
$$

Therefore, we have by (2.37), (2.36) and (2.33),

$$
\begin{aligned}
& \sum_{j+|x|=k}\left\|\left(1+\frac{|x|}{t}\right)^{-j} \partial_{x}^{\alpha} \partial_{t}^{j} \int_{\mathbf{R}^{3}} \frac{1}{|y|}\left|\hat{\psi}_{+0}\left(\frac{x}{t}-y\right)\right|^{2} d y\right\|_{L^{x}} \\
& \leqq C t^{-k} \sum_{\left|\alpha_{1}\right|+\left|\alpha_{2}\right|=k}\left\|\int_{\mathbf{R}^{3}} \frac{1}{|y|}\left|\left(\partial_{x}^{\alpha_{1}} \hat{\psi}_{+0}\right)\left(\frac{x}{t}-y\right)\right|\left|\left(\partial_{x}^{\alpha_{2}} \overline{\hat{\psi}}_{+0}\right)\left(\frac{x}{t}-y\right)\right| d y\right\|_{L^{\infty}}
\end{aligned}
$$




$$
\begin{aligned}
\leqq & C t^{-k} \sum_{\left|\alpha_{1}\right|+\left|\alpha_{2}\right|=k}\left\|\int_{\mathbf{R}^{3}} \frac{1}{|y|}\left|\partial_{x}^{\alpha_{1}} \hat{\psi}_{+0}(x-y)\right|\left|\partial_{x}^{\alpha_{2}} \overline{\hat{\psi}}_{+0}(x-y)\right| d y\right\|_{L^{\alpha}} \\
\leqq & C t^{-k} \sum_{\left|\alpha_{1}\right|+\left|\alpha_{2}\right|=k}\left\|\left(\partial_{x}^{\alpha_{1}} \hat{\psi}_{+0}\right)\left(\partial_{x}^{\alpha_{2}} \overline{\hat{\psi}}_{+0}\right)\right\|_{L^{2}}^{1 / 2}\left\|\left(\partial_{x}^{\alpha_{1}} \hat{\psi}_{+0}\right)\left(\partial_{x}^{\alpha_{2}} \overline{\hat{\psi}}_{+0}\right)\right\|_{L^{6 / 5}}^{1 / 2} \\
\leqq & C t^{-k} \sum_{\left|\alpha_{1}\right|+\left|\alpha_{2}\right|=k}\left\|\partial_{x}^{\alpha_{1}} \hat{\psi}_{+0}\right\|_{L^{\infty}}^{1 / 2}\left\|\partial_{x}^{\alpha_{2}} \overline{\hat{\psi}}_{+0}\right\|_{L^{2}}^{1 / 2} \\
& \times\left\|\partial_{x}^{\alpha_{1}} \hat{\psi}_{+0}\right\|_{L^{\infty}}^{1 / 6}\left\|\partial_{x}^{\alpha_{1}} \hat{\psi}_{+0}\right\|_{L^{2}}^{1 / 3}\left\|\partial_{x}^{\alpha_{2}} \overline{\hat{\psi}}_{+0}\right\|_{L^{2}}^{1 / 2} \\
\leqq & C t^{-k}\left\|\hat{\psi}_{+0}\right\|_{W^{k, \infty}}^{2 / 3}\left\|\hat{\psi}_{+0}\right\|_{H^{k}}^{4 / 3} \\
\leqq & C t^{-k}\left\|\psi \psi_{+0}\right\|_{H^{6,7}}^{2}, \quad t \geqq 1 .
\end{aligned}
$$

On the other hand, let $k$ be any integer with $0 \leqq k \leqq 5$ and let $j+|\alpha|=k$. If $1 \leqq k \leqq 5$ and $j \neq 0$, we have

$$
\begin{aligned}
\left|\partial_{x}^{\alpha} \partial_{t}^{j} \int_{0}^{t} R\left(s, \frac{s}{t} x\right) d s\right| \leqq & C \sum_{j+|\alpha|=k-1}\left|\partial_{x}^{\alpha} \partial_{t}^{j} R(t, x)\right| \\
& +C t^{-k}\left(1+\frac{|x|}{t}\right)^{j} \sum_{|\alpha|=k} \int_{0}^{t} s^{k}\left|\left(\partial_{x}^{\alpha} R\right)\left(s, \frac{s}{t} x\right)\right| d s, \quad t \geqq 1 .
\end{aligned}
$$

If $0 \leqq k \leqq 5$ and $j=0$, we have

$$
\sum_{|\alpha|=k}\left|\partial_{x}^{\alpha} \int_{0}^{t} R\left(s, \frac{s}{t} x\right) d s\right| \leqq C t^{-k} \sum_{|\alpha|=k} \int_{0}^{t} s^{k}\left|\left(\partial_{x}^{\alpha} R\right)\left(s, \frac{s}{t} x\right)\right| d s, \quad t \geqq 1 .
$$

Therefore, (2.31), (2.39) and (2.40) yield

$$
\begin{aligned}
\sum_{k=0}^{5} & \sum_{j+|x|=k}(1+t)^{k}(\log (1+t))^{-1}\left\|\left(1+\frac{|x|}{t}\right)^{-j} \partial_{x}^{\alpha} \partial_{t}^{j} \int_{0}^{t} R\left(s, \frac{s}{t} x\right) d s\right\|_{L^{\infty}} \\
\leqq & C \sum_{k=1}^{5} \sum_{j+|\alpha|=k}(1+t)^{k}(\log (1+t))^{-1}\left\|\left(1+\frac{|x|}{t}\right)^{-j} \partial_{x}^{\alpha} \partial_{t}^{j} R(t)\right\|_{L^{\infty}} \\
& +C \sum_{k=1}^{5} \sum_{|x|=k}(1+t)^{k} t^{-k}(\log (1+t))^{-1} \int_{0}^{t} s^{k}\left\|\left(\partial_{x}^{\alpha} R\right)\left(s, \frac{s}{t} x\right)\right\|_{L^{\infty}} d s \\
& +C \sum_{k=0}^{5} \sum_{|\alpha|=k}(1+t)^{k} t^{-k}(\log (1+t))^{-1} \int_{0}^{t} s^{k}\left\|\left(\partial_{x}^{\alpha} R\right)\left(s, \frac{s}{t} x\right)\right\|_{L^{\infty}} d s \\
\leqq & C \sum_{k=1}^{5}(1+t)^{k}(\log (1+t))^{-1}(1+t)^{-k}\|\psi+0\|_{H^{6,7}}^{2} \\
& +C \sum_{k=1}^{5}(\log (1+t))^{-1} \int_{0}^{t} s^{k}(1+t)^{-k-1} d s\|\psi+0\|_{H^{6,7}}^{2} \\
& +C \sum_{k=0}^{5}(\log (1+t))^{-1} \int_{0}^{t} s^{k}(1+t)^{-k-1} d s\|\psi+0\|_{H^{6,7}}^{2} \\
\leqq & C\|\psi+0\|_{H^{6,7}}^{2}, t \geqq 1 .
\end{aligned}
$$


Equation (2.32) follows immediately from (2.38), (2.41) and the definition of $\varphi(t, x)$.

The following corollary is an immediate consequence of Lemmas 2.1(iii), 2.2(ii) and 2.4 .

Corollary 2.5. Let $\delta>0$ and let all the assumptions in Theorem 1.1 be satisfied. We put

$$
m(t, x)=(i t)^{-3 / 2} e^{\frac{i|x|^{2}}{2 t}} \hat{\psi}_{+0}\left(\frac{x}{t}\right) \theta(t) .
$$

Let $\psi_{+}(t), A_{+}(t)$ and $\varphi(t, x)$ be defined as in Theorem 1.1 .

(i) The following relations hold.

$$
\begin{gathered}
\sum_{j=0}^{1}\left\|\partial_{t}^{j}\left\{e^{i \varphi} \psi_{+}(t)-e^{i \varphi} m(t)\right\}\right\|_{H^{3-2 \jmath}}=O\left(t^{-1}\right) \quad(t \rightarrow \infty), \\
\left(\int_{t}^{\infty} \sum_{2 j+|\alpha| \leqq 2}\left\|\partial_{x}^{\alpha} \partial_{s}^{j}\left\{e^{i \varphi} \psi_{+}(s)-e^{i \varphi} m(s)\right\}\right\|_{L^{4}}^{4} d s\right)^{1 / 4}=O\left(t^{-3 / 2}\right) \quad(t \rightarrow \infty) .
\end{gathered}
$$

(ii) There exists a $K_{8}>0$ such that

$$
\begin{aligned}
& \sum_{2 \jmath+|x| \leqq 3}\left\|\partial_{x}^{\alpha} \partial_{t}^{j}\left\{e^{i \varphi} A_{+}(t) m(t)\right\}\right\|_{L^{2}} \\
& \leqq \\
& \quad K_{8}(1+t)^{-2}\left(1+\left\|\psi_{+0}\right\|_{H^{6,7}}^{2}\right)^{3}\left(\left\|A_{+0}\right\|_{\dot{H}^{5} \cap \dot{H}^{1}}+\left\|A_{+0}\right\|_{W^{5,1}}\right. \\
& \left.\quad+\left\|A_{+1}\right\|_{H^{4}}+\left\|A_{+1}\right\|_{W^{4,1}}\right)\left\|\psi_{+0}\right\|_{H^{6,7}}, \quad t \geqq 0,
\end{aligned}
$$

where $K_{8}$ depends only on $\delta$.

Proof. We first show (i). Equation (2.42) follows directly from Lemma 2.1 (iii) and Lemma 2.4. On the other hand, (2.3), (2.4), the interpolation and Lemma 2.4 yield

$$
\sum_{2 j+|x| \leqq 2}\left\|\partial_{t}^{\alpha} \partial_{t}^{j}\left\{e^{i \varphi} \psi_{+}(t)-e^{i \varphi} m(t)\right\}\right\|_{L^{4}} \leqq C t^{-7 / 4}, \quad t \geqq 1,
$$

which implies (2.43).

We next show (ii). Since

$$
\operatorname{supp} m(t, x) \subset\left\{x \in \mathbf{R}^{3} ;|x| \geqq(1+\delta) t\right\} \cup\left\{x \in \mathbf{R}^{3} ;|x| \leqq(1-\delta) t\right\}
$$

for each $t>0$, Lemma 2.2(ii) and Lemma 2.4 give us (2.44).

Remark 2.2. The assumption on the support of $\hat{\psi}_{+_{0}}$ is not needed for the proof of Corollary 2.5(i), but it is indispensable to the proof of Corollary 2.5(ii).

We conclude this section by giving the following lemma.

Lemma 2.6. Let $s$ be a real number with $s>1$ and let $k$ be a positive integer. Then, for some $C_{s k}>0$,

$$
\int_{t}^{\infty}(1+\tau)^{-s}(\log (2+\tau))^{k} d \tau \leqq C_{s k}(1+t)^{-s+1}(\log (2+t))^{k}, \quad t \geqq 0,
$$

where $C_{\text {sk }}$ depends only on $s$ and $k$. Furthermore, we can choose $C_{s k}=5$ in (2.45) for $(s, k)=(4,3),(3,4),(7 / 2,3)$ and $(2,2)$.

Lemma 2.6 follows immediately from the integration by parts. 


\section{Proof of Theorem 1.1}

In this section we prove Theorem 1.1 by using the results obtained in Sect. 2 .

As stated in Sect. 1, we use (1.2) to rewrite (1.1) and (1.2) as (1.14) and (1.15). We next put

$$
\begin{aligned}
& A(t)=A_{+}(t)+R(t)+v(t), \\
& \psi(t)=e^{i \varphi} m(t)+u(t)
\end{aligned}
$$

where $A_{+}(t), R(t)$ and $\varphi(t, x)$ are defined in Theorem 1.1 and

$$
m(t, x)=(i t)^{-3 / 2} e^{\frac{l|x|^{2}}{2 t}} \hat{\psi}_{+0}\left(\frac{x}{t}\right) \theta(t) .
$$

We substitute (3.1) and (3.2) into (1.14) and (1.15) to obtain

$$
\begin{gathered}
\partial_{t}^{2} v-\Delta v=f(t)+F(t, v, u), \quad t>0, \quad x \in \mathbf{R}^{3}, \\
2 i \partial_{t} u+\Delta u=2 i\left(A_{+}+R+v\right) \cdot \nabla u \\
+g(t)+G_{1}(t, v, u)+G_{2}(t, v, u), \quad t>0, \quad x \in \mathbf{R}^{3}, \\
\left\|\partial_{t} v(t)\right\|_{L^{2}}^{2}+\sum_{j=1}^{3}\left\|\nabla v_{j}\right\|_{L^{2}}^{2} \rightarrow 0 \quad(t \rightarrow \infty), \\
\|u(t)\|_{L^{2}} \rightarrow 0 \quad(t \rightarrow \infty) .
\end{gathered}
$$

Here

$$
\begin{gathered}
f(t)=f_{0}(t)+\frac{1}{4 \pi} \nabla\left\{\frac{1}{|x|} *\left(\nabla \cdot f_{0}(t)\right)\right\} \\
F(t, v, u)=F_{0}(t, v, u)+\frac{1}{4 \pi} \nabla\left\{\frac{1}{|x|} *\left(\nabla \cdot F_{0}(t, v, u)\right)\right\} \\
g(t)=-i \Delta \varphi e^{i \varphi} m+|\nabla \varphi|^{2} e^{i \varphi} m-i e^{i \varphi} \frac{1}{t} \nabla \varphi \cdot\left\{(i t)^{-3 / 2} e^{\frac{i|x|^{2}}{2 t}}\left(\nabla \hat{\psi}_{+0}\right)\left(\frac{x}{t}\right) \theta(t)\right\} \\
-i e^{i \varphi} \frac{1}{t^{2}}(i t)^{-3 / 2} e^{\frac{i|x|^{2}}{2 t}}\left(\Delta \hat{\psi}_{+0}\right)\left(\frac{x}{t}\right) \theta(t) \\
+i \operatorname{div}(A++R) e^{i \varphi} m-2 e^{i \varphi} A+\cdot\left(\frac{x}{t} m\right)-2 e^{i \varphi}(A++R) \cdot \nabla \varphi m \\
+2 i e^{i \varphi} \frac{1}{t}(A++R) \cdot\left\{(i t)^{-3 / 2} e^{\frac{i|x|^{2}}{2 t}}\left(\nabla \hat{\psi}_{+0}\right)\left(\frac{x}{t}\right) \theta(t)\right\} \\
+|A++R|^{2} e^{i \varphi} m-i e^{i \varphi}(i t)^{-3 / 2} e^{\frac{i|x|^{2}}{2 t}} \hat{\psi}_{+0}\left(\frac{x}{t}\right) \frac{d}{d t} \theta(t) \\
G_{1}(t, v, u)=i \operatorname{div}(A++R+v) u-2 e^{i \varphi} v \cdot\left(\frac{x}{t} m\right)-2 e^{i \varphi} v \cdot \nabla \varphi m
\end{gathered}
$$


Global Existence and Asymptotic Behavior of Solutions

$$
\begin{aligned}
+ & 2 i e^{i \varphi} \frac{1}{t} v \cdot\left\{(i t)^{-3 / 2} e^{\frac{i|x|^{2}}{2 t}}(\nabla \hat{\psi}+0)\left(\frac{x}{t}\right) \theta(t)\right\} \\
+ & 2\left(A_{+}+R\right) \cdot v e^{i \varphi} m+2\left(A_{+}+R\right) \cdot v u \\
+ & 2|v|^{2} e^{i \varphi} m+2|v|^{2} u \\
G_{2}(t, v, u)= & \frac{1}{2 \pi}\left\{\frac{1}{|x|} * \mathfrak{R}\left(e^{i \varphi} m \bar{u}\right)\right\} e^{i \varphi} m \\
& +\frac{1}{4 \pi}\left\{\frac{1}{|x|} *|m|^{2}\right\} u+\frac{1}{4 \pi}\left\{\frac{1}{|x|} *|u|^{2}\right\} e^{i \varphi} m \\
& +\frac{1}{2 \pi}\left\{\frac{1}{|x|} * \mathfrak{R}\left(e^{i \varphi} m \bar{u}\right)\right\} u+\frac{1}{4 \pi}\left\{\frac{1}{|x|} *|u|^{2}\right\} u,
\end{aligned}
$$

where

$$
\begin{gathered}
f_{0}(t)=2\left(\nabla-A_{+}-R\right)|m|^{2}, \\
F_{0}(t, v, u)=2 \mathfrak{J}\left(e^{-i \varphi} \bar{m} \nabla u\right)+2 \nabla \varphi \Re\left(e^{i \varphi} m \bar{u}\right) \\
+2 \mathfrak{J}\left(e^{i \varphi} \nabla m \bar{u}\right)+2 \mathfrak{I}(\bar{u} \nabla u)-2 v|m|^{2} \\
-4\left(A_{+}+R+v\right) \mathfrak{R}\left(e^{i \varphi} m \bar{u}\right)-2\left(A_{+}+R+v\right)|u|^{2} .
\end{gathered}
$$

We shall solve (3.4)-(3.7) by the contraction mapping principle. Let $\eta$ be a positive constant satisfying (1.4). By Lemmas 2.2, 2.4, Corollary 2.5(ii) and (2.35), we obtain

$$
\begin{gathered}
\sum_{j=0}^{1} \sum_{|\alpha| \leqq 2-j}\left\|\partial_{x}^{\alpha} \partial_{t}^{j} f(t)\right\|_{L^{2}} \leqq C(1+\eta)^{2} \eta^{2}(1+t)^{-5 / 2} \log (2+t), \quad t \geqq 0, \\
\sum_{2 j+|\alpha| \leqq 3}\left\|\partial_{x}^{\alpha} \partial_{t}^{j} g(t)\right\|_{L^{2}} \leqq C(1+\eta)^{10} \eta(1+t)^{-2}(\log (2+t))^{2}, \quad t \geqq 0,
\end{gathered}
$$

where $C$ does not depend on $\eta$. Therefore, if we choose $\eta>0$ such that

$$
\eta \leqq 1
$$

we have by (3.8) and (3.9)

$$
\begin{gathered}
\left(\sum_{j=0}^{1} \sum_{|\alpha| \leqq 2}\left\|\partial_{x}^{\alpha} \partial_{t}^{j} f(t)\right\|_{L^{2}}^{2}\right)^{1 / 2} \leqq M \eta(1+t)^{-5 / 2} \log (2+t), \quad t \geqq 0, \\
\max \left\{\left(\sum_{j+|\alpha| \leqq 3}\left\|\partial_{x}^{\alpha} \partial_{t}^{j} g(t)\right\|_{L^{2}}^{2}\right)^{1 / 2}, \sum_{j+|\alpha| \leqq 3}\left\|\partial_{x}^{\alpha} \partial_{t}^{j} g(t)\right\|_{L^{2}}\right\} \\
\leqq M \eta(1+t)^{-2}(\log (2+t))^{2}, \quad t \geqq 0
\end{gathered}
$$

for some $M \geqq 0$, where $M$ is independent of $\eta$ for $0<\eta \leqq 1$. We put

$$
\begin{aligned}
X=\left\{[v(t), u(t)] ; v(t) \in C\left([0, \infty) ; \dot{H}^{3} \cap \dot{H}^{1}\right),\right. \\
\\
\partial_{t} v(t) \in C\left([0, \infty) ; H^{2}\right),
\end{aligned}
$$




$$
\begin{aligned}
& u(t) \in \bigcap_{j=0}^{1} C^{j}\left([0, \infty) ; H^{3-2 j}\right), \\
& \sup _{t \geqq 0}\left[( 1 + t ) ^ { 3 / 2 } ( \operatorname { l o g } ( 2 + t ) ) ^ { - 2 } \left(\sum_{1 \leqq|\alpha| \leqq 2}\left\|\partial_{x}^{\alpha} v(t)\right\|_{L^{2}}^{2}\right.\right. \\
& \left.\left.+\sum_{|x| \leqq 1}\left\|\partial_{x}^{\alpha} \partial_{t} v(t)\right\|_{L^{2}}^{2}\right)^{1 / 2}\right] \leqq 11 M \eta \\
& \sup _{t \geqq 0}\left[( 1 + t ) ( \operatorname { l o g } ( 2 + t ) ) ^ { - 2 } \left(\sum_{|x|=3}\left\|\partial_{x}^{\alpha} v(t)\right\|_{L^{2}}^{2}\right.\right. \\
& \left.\left.+\sum_{|x|=2}\left\|\partial_{x}^{\alpha} \partial_{t} v(t)\right\|_{L^{2}}^{2}\right)^{1 / 2}\right] \leqq 11 M \eta, \\
& \sup _{t \geqq 0}\left[(1+t)(\log (2+t))^{-2}\left(\sum_{|\alpha| \leqq 2}\left\|\partial_{x}^{\alpha} u(t)\right\|_{L^{2}}^{2}\right)^{1 / 2}\right] \leqq 6 M \eta, \\
& \sup _{t \geqq 0}\left[(1+t)(\log (2+t))^{-2}\left(\sum_{|\alpha| \leqq 1}\left\|\partial_{x}^{\alpha} \partial_{t} u(t)\right\|_{L^{2}}^{2}\right)^{1 / 2}\right] \leqq 21 M \eta, \\
& \left.\sup _{t \geqq 0}\left[(1+t)(\log (2+t))^{-2} \sum_{|\alpha| \leqq 2}\left(\int_{t}^{\infty}\left\|\partial_{x}^{\alpha} u(s)\right\|_{L^{4}}^{8 / 3} d s\right)^{3 / 8}\right] \leqq 6 K_{1} M \eta\right\} \\
& \sup _{t \geqq 0}\left[(1+t)(\log (2+t))^{-2} \sum_{|\alpha|=1}\left\|\Delta \partial_{x}^{\alpha} u(t)\right\|_{L^{2}}\right] \leqq 3(14 \sqrt{3}+1) M \eta,
\end{aligned}
$$

where $K_{1}$ is defined in Lemma 2.1(ii). We introduce the metric $d\left(\left[v_{1}, u_{1}\right],\left[v_{2}, u_{2}\right]\right)$ into $X$ as follows:

$$
\begin{aligned}
& d\left(\left[v_{1}, u_{1}\right],\left[v_{2}, u_{2}\right]\right)=\sum_{1 \leqq|\alpha| \leqq 2} \sup _{t \geqq 0}\left[(1+t)^{3 / 2}(\log (2+t))^{-2}\left\|\partial_{x}^{\alpha}\left(v_{1}(t)-v_{2}(t)\right)\right\|_{L^{2}}\right] \\
& \quad+\sum_{|x| \leqq 1} \sup _{t \geqq 0}\left[(1+t)^{3 / 2}(\log (2+t))^{-2}\left\|\partial_{x}^{\alpha} \partial_{t}\left(v_{1}(t)-v_{2}(t)\right)\right\|_{L^{2}}\right] \\
& \quad+\sum_{|\alpha|=3} \sup _{t \geqq 0}\left[(1+t)(\log (2+t))^{-2}\left\|\partial_{x}^{\alpha}\left(v_{1}(t)-v_{2}(t)\right)\right\|_{L^{2}}\right] \\
& \quad+\sum_{|\alpha|=2} \sup _{t \geqq 0}\left[(1+t)(\log (2+t))^{-2}\left\|\partial_{x}^{\alpha} \partial_{t}\left(v_{1}(t)-v_{2}(t)\right)\right\|_{L^{2}}\right] \\
& \quad+\sum_{2 J+|\alpha| \leqq 3} \sup _{t \geqq 0}\left[(1+t)(\log (2+t))^{-2}\left\|\partial_{x}^{\alpha} \partial_{t}\left(u_{1}(t)-u_{2}(t)\right)\right\|_{L^{2}}\right] \\
& \quad+\sum_{|x| \leqq 2} \sup _{t \geqq 0}\left[(1+t)(\log (2+t))^{-2}\left(\int_{t}^{\infty}\left\|\partial_{x}^{\alpha}\left(u_{1}(s)-u_{2}(s)\right)\right\|_{L^{4}}^{8 / 3} d s\right)^{3 / 8}\right]
\end{aligned}
$$

for $\left[v_{1}, u_{1}\right],\left[v_{2}, u_{2}\right] \in X$. We note that $X$ is a complete metric space with the metric $d$. We first define the nonlinear mapping $N_{1}[v, u]$ for $[v, u] \in X$. For $[v, u] \in X$, we 
consider the linear problem:

$$
\begin{gathered}
\partial_{t}^{2} w-\Delta w=f(t)+F(t, v, u), \quad t \geqq 0, \quad x \in \mathbf{R}^{3}, \\
\sum_{j=1}^{3}\left\|\nabla w_{j}(t)\right\|_{L^{2}}^{2}+\left\|\hat{\partial}_{t} w(t)\right\|_{L^{2}}^{2} \rightarrow 0 \quad(t \rightarrow \infty) .
\end{gathered}
$$

Let $N_{1}[v, u]$ be a mapping from $[v, u] \in X$ to a solution $w(t)=\left(w_{1}(t), w_{2}(t), w_{3}(t)\right)$ of (3.13)-(3.14). We next define the nonlinear mapping $N_{2}[v, u]$ as follows. For $[v, u] \in X$, we consider the following linear problem:

$$
\begin{aligned}
& 2 i \partial_{t} z+\Delta z= 2 i\left(A_{+}+R+v\right) \cdot \nabla z \\
&+g(t)+G_{1}(t, v, u)+G_{2}(t, v, u), \quad t \geqq 0, \quad x \in \mathbf{R}^{3}, \\
&\|z(t)\|_{L^{2}} \rightarrow 0 \quad(t \rightarrow \infty) .
\end{aligned}
$$

Let $N_{2}[v, u]$ be a mapping from $[v, u] \in X$ to a solution $z(t)$ of (3.15)-(3.16). We put $N[v, u]=\left[N_{1}[v, u], N_{2}[v, u]\right]$. By the definition of $N$, we see that the fixed point $[u, v]$ of the nonlinear mapping $N$ in $X$ are the solutions of (3.4)-(3.7). Now we show that if $\eta>0$ is sufficiently small, the nonlinear mapping $N$ is a contraction from $X$ into $X$.

We first evaluate $w=N_{1}[v, u]$. By Lemmas 2.2(i), 2.4 and the GagliardoNirenberg inequality (see, e.g., [4]) we have

$$
\begin{aligned}
\sum_{|x| \leqq 1} \| & \partial_{x}^{\alpha} F_{0}(t, v, u) \|_{L^{2}} \\
\leqq & C \sum_{|x| \leq 1}\left[\left\|\partial_{x}^{\alpha}\left\{\mathfrak{J}\left(e^{-l \varphi} \bar{m} \nabla u\right)+\nabla \varphi \mathfrak{R}\left(e^{i \varphi} m \bar{u}\right)+\mathfrak{J}\left(e^{i \varphi} \nabla m \bar{u}\right)\right\}\right\|_{L^{2}}\right. \\
& +\left\|\partial_{x}^{\alpha}(\bar{u} \nabla u)\right\|_{L^{2}}+\left\|\partial_{x}^{\alpha}\left(v|m|^{2}\right)\right\|_{L^{2}}+\left\|\partial_{x}^{\alpha}\left\{\left(A_{+}+R+v\right) \Re\left(e^{i \varphi} m \bar{u}\right)\right\}\right\|_{L^{2}} \\
& \left.+\left\|\partial_{x}^{\alpha}\left\{(A++R+v)|u|^{2}\right\}\right\|_{L^{2}}\right] \\
\leqq & C\left(1+\|\nabla \varphi(t)\|_{W^{1, \infty}}\right)^{3}\|m(t)\|_{W^{2, \infty}}\|u(t)\|_{H^{1}} \\
& +C\left(\|u(t)\|_{W^{1,4}}\|\nabla u(t)\|_{L^{4}}+\|u(t)\|_{L^{4}}\|\nabla u(t)\|_{W^{1,4}}\right) \\
& +C\left(\|v(t)\|_{L^{\infty}}\|m(t)\|_{W^{1, \infty}}\|m(t)\|_{H^{1}}+\|v(t)\|_{\dot{H}^{1}}\|m(t)\|_{L^{\infty}}^{2}\right) \\
& +C\left(\|A+(t)\|_{W^{1, \infty}}+\|R(t)\|_{W^{1, \infty}}\right)\|m(t)\|_{W^{1, \infty}}\|u(t)\|_{H^{1}} \\
& +C\left(\|v(t)\|_{L^{\infty}}\|m(t)\|_{W^{1, \infty}}\|u(t)\|_{H^{1}}+\|v(t)\|_{\dot{H}^{1}}\|m(t)\|_{L^{\infty}}\|u(t)\|_{L^{\infty}}\right) \\
& +C\left(\|A+(t)\|_{W^{1, \infty}}+\|R(t)\|_{W^{1, \infty}}\right)\|u(t)\|_{W^{1,4}}^{2} \\
& +C\left(\|v(t)\|_{L^{\infty}}\|u(t)\|_{W^{1,4}}^{2}+\|v(t)\|_{\dot{H}^{1}}\|u(t)\|_{L^{\infty}}^{2}\right) \\
\leqq & C\left(1+\eta^{2}\right)^{3} \eta^{2}(1+t)^{-5 / 2}(\log (2+t))^{2}+C\|u(t)\|_{W^{2,4}}^{2} \\
& +C \eta^{2}(1+t)^{-3 / 2}\|v(t)\|_{\dot{H}^{2}}^{1 / 2}\|v(t)\|_{\dot{H}^{1}}^{1 / 2} \\
& +C \eta^{3}(1+t)^{-4}(\log (2+t))^{2}+C(1+\eta) \eta^{2}(1+t)^{-3}(\log (2+t))^{2} \\
& +C \eta^{2}(1+t)^{-5 / 2}(\log (2+t))^{2}\|v(t)\|_{\dot{H}^{2}}^{1 / 2}\|v(t)\|_{\dot{H}^{1}}^{1 / 2}
\end{aligned}
$$




$$
\begin{aligned}
& +C \eta^{3}(1+t)^{-7 / 2}(\log (2+t))^{4}+C(1+\eta) \eta(1+t)^{-1}\|u(t)\|_{W^{1,4}}^{2} \\
& +C\|v(t)\|_{\dot{H}^{2}}^{1 / 2}\|v(t)\|_{\dot{H}^{1}}^{1 / 2}\|u(t)\|_{W^{1,4}}^{2} \\
& +C \eta(1+t)^{-3 / 2}(\log (2+t))^{2}\|u(t)\|_{\dot{H}^{2}}\|u(t)\|_{\dot{H}^{1}} \\
\leqq & C(1+\eta)^{6} \eta^{2}(1+t)^{-5 / 2}(\log (2+t))^{2}+C(1+\eta)^{2}\|u(t)\|_{W^{2,4}}^{2}, \quad t \geqq 0
\end{aligned}
$$

for $[v, u] \in X$. In the same way as above, we have

$$
\begin{aligned}
\sum_{|\alpha|=2}\left\|\partial_{x}^{\alpha} F_{0}(t, v, u)\right\|_{L^{2}} \leqq & C(1+\eta)^{6} \eta^{2}(1+t)^{-5 / 2}(\log (2+t))^{2}+C\|u(t)\|_{W^{2,4}}^{2} \\
& +C \eta(1+t)^{-1}(\log (2+t))^{2}\|u(t)\|_{W^{1,4}}, \quad t \geqq 0
\end{aligned}
$$

for $[v, u] \in X$.

We consider the regularized problem associated with (3.13)-(3.14):

$$
\begin{gathered}
\partial_{t}^{2} w_{\varepsilon \lambda}-\Delta w_{\varepsilon \lambda}=(1+\lambda t)^{-4} \rho_{\varepsilon} *\{f(t)+F(t, v, u)\}, \quad t \geqq 0, \quad x \in \mathbf{R}^{3}, \\
\sum_{j=1}^{3}\left\|\nabla w_{\varepsilon \lambda j}(t)\right\|_{L^{2}}^{2}+\left\|\partial_{t} w_{\varepsilon \lambda}(t)\right\|_{L^{2}}^{2} \rightarrow 0 \quad(t \rightarrow \infty),
\end{gathered}
$$

where $0<\lambda<1$ and $0<\varepsilon<1$. The solution $w_{\varepsilon \hat{\lambda}}(t)$ of (3.19)-(3.20) is given by $w_{\varepsilon \hat{\lambda}}(t)=-\int_{t}^{\infty}(1+\lambda s)^{-4}\left(\omega^{-1} \sin \omega(t-s)\right) \rho_{\varepsilon} *\{f(s)+F(s, v, u)\} d s, \quad t \geqq 0$.

Formula (3.21) and a direct calculation yield

$$
\begin{gathered}
w_{\varepsilon \lambda}(t) \in \bigcap_{j=1}^{\infty} C^{2}\left([0, \infty) ; H^{j}\right), \\
\sum_{j=1}^{2} \sum_{|\alpha| \leqq 5-j} \sup _{t \geqq 0}\left[(1+\lambda t)^{4}\left\|\partial_{x}^{\alpha} \partial_{t}^{j} w_{\varepsilon \lambda}(t)\right\|_{L^{2}}+(1+\lambda t)^{3}\left\|w_{\varepsilon \lambda}(t)\right\|_{L^{2}}\right]<+\infty
\end{gathered}
$$

for $0<\lambda<1$ and $0<\varepsilon<1$.

We next derive the a priori estimates independent of $\varepsilon$ and $\lambda$ for $w_{\varepsilon \lambda}$. Let $\alpha$ be an arbitrary multi-index with $|\alpha| \leqq 1$. We apply $\partial_{x}^{x}$ to (3.19) and take the scalar product in $L^{2}$ between the resulting equation and $\partial_{t} \partial_{x}^{\alpha} w_{\varepsilon \lambda}(t)$ to obtain

$$
\begin{aligned}
& \frac{1}{2} \frac{d}{d t}\left\{\left\|\partial_{t} \partial_{x}^{\alpha} w_{\varepsilon \lambda}(t)\right\|_{L^{2}}^{2}+\sum_{j=1}^{3}\left\|\nabla \partial_{x}^{\alpha} w_{\varepsilon \lambda j}(t)\right\|_{L^{2}}^{2}\right\} \\
& \quad=(1+\lambda t)^{-4}\left(\rho_{\varepsilon} *\left\{\partial_{x}^{\alpha} f(t)+\partial_{x}^{\alpha} F(t, v, u)\right\}, \partial_{t} \partial_{x}^{\alpha} w_{\varepsilon \lambda}(t)\right), \quad t \geqq 0 .
\end{aligned}
$$

Integrating (3.24) over $[t, \infty)$, we have by (3.11), (3.17), (2.36) and (3.23)

$$
\begin{aligned}
& \left\|\partial_{t} \partial_{x}^{\alpha} w_{\varepsilon \lambda}(t)\right\|_{L^{2}}^{2}+\sum_{j=1}^{3}\left\|\nabla \partial_{x}^{\alpha} w_{\varepsilon \lambda j}(t)\right\|_{L^{2}}^{2} \\
& =-2 \int_{t}^{\infty}(1+\lambda s)^{-4}\left(\rho_{\varepsilon} *\left\{\partial_{x}^{\alpha} f(s)+\partial_{x}^{\alpha} F(s, v, u)\right\}, \partial_{s} \partial_{x}^{\alpha} w_{\varepsilon \lambda}(s)\right) d s \\
& \leqq 2 \int_{t}^{\infty}\left\{\left\|\partial_{x}^{\alpha} f(s)\right\|_{L^{2}}+\left\|\partial_{x}^{\alpha} F(s, v, u)\right\|_{L^{2}}\right\}\left\|\partial_{s} \partial_{x}^{\alpha} w_{\varepsilon \lambda}(s)\right\|_{L^{2}} d s .
\end{aligned}
$$


Since $\alpha$ is an arbitrary multi-index with $|\alpha| \leqq 1$, we take the summation of (3.25) over $|\alpha| \leqq 1$ to obtain

$$
\begin{aligned}
& \sum_{|x| \leqq 1}\left\|\partial_{x}^{\alpha} \partial_{t} w_{\varepsilon \lambda}(t)\right\|_{L^{2}}^{2}+\sum_{1 \leqq|\alpha| \leqq 2}\left\|\partial_{x}^{\alpha} w_{\varepsilon \lambda}(t)\right\|_{L^{2}}^{2} \\
& \leqq 2 \sum_{|x| \leqq 1} \int_{t}^{\infty}\left\{\left\|\partial_{x}^{\alpha} f(s)\right\|_{L^{2}}+\left\|\partial_{x}^{\alpha} F(s, v, u)\right\|_{L^{2}}\right\}\left\|\partial_{x}^{\alpha} \partial_{s} w_{\varepsilon \lambda}(s)\right\|_{L^{2}} d s \\
& \leqq \\
& \quad \int_{t}^{\infty}\left\{\left(\sum_{|x| \leqq 1}\left\|\partial_{x}^{\alpha} f(s)\right\|_{L^{2}}^{2}\right)^{1 / 2}+\left(\sum_{|x| \leqq 1}\left\|\partial_{x}^{\alpha} F(s, v, u)\right\|_{L^{2}}^{2}\right)^{1 / 2}\right\} \\
& \quad \times\left(\sum_{|\alpha| \leqq 1}\left\|\partial_{x}^{\alpha} \partial_{s} w_{\varepsilon \lambda}(s)\right\|_{L^{2}}^{2}\right)^{1 / 2} d s, \quad t \geqq 0 .
\end{aligned}
$$

At the last inequality of (3.26) we have used the Hölder inequality for sequence. Equations (3.11), (3.17), (2.36), (3.26) and Lemma 2.6 yield

$$
\begin{aligned}
& \sum_{|x| \leqq 1}\left\|\partial_{x}^{\alpha} \partial_{t} w_{\varepsilon \lambda}(t)\right\|_{L^{2}}^{2}+\sum_{1 \leqq|\alpha| \leqq 2}\left\|\partial_{x}^{\alpha} w_{\varepsilon \lambda}(t)\right\|_{L^{2}}^{2} \\
& \leqq 2 \int_{t}^{\infty} M \eta(1+s)^{-4}(\log (2+s))^{3} d s \\
& \times \sup _{t \geqq 0}\left[(1+t)^{3 / 2}(\log (2+t))^{-2}\left(\sum_{|\gamma| \leqq 1}\left\|\partial_{x}^{\alpha} \partial_{t} w_{\varepsilon \hat{\lambda}}(t)\right\|_{L^{2}}^{2}\right)^{1 / 2}\right] \\
& +C \int_{t}^{\infty}\left\{(1+\eta)^{6} \eta^{2}(1+s)^{-4}(\log (2+s))^{2}\right.
\end{aligned}
$$

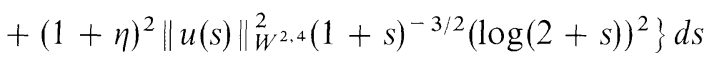

$$
\begin{aligned}
& \times \sup _{t \geqq 0}\left[(1+t)^{3 / 2}(\log (2+t))^{-2}\left(\sum_{|\gamma| \leqq 1}\left\|\partial_{x}^{\alpha} \partial_{t} w_{\varepsilon \lambda}(t)\right\|_{L^{2}}^{2}\right)^{1 / 2}\right] \\
& \leqq\left\{10 M \eta(1+t)^{-3}(\log (2+t))^{4}+C(1+\eta)^{6} \eta^{2}(1+t)^{-3}(\log (2+t))^{4}\right. \\
& \left.+C(1+\eta)^{2}\left(\int_{t}^{\infty}\|u(s)\|_{W^{2,4}}^{8 / 3} d s\right)^{3 / 4}\left(\int_{t}^{\infty}(1+s)^{-6}(\log (2+s))^{8} d s\right)^{1 / 4}\right\} \\
& \times \sup _{t \geqq 0}\left[(1+t)^{3 / 2}(\log (2+t))^{-2}\left(\sum_{|x| \leqq 1}\left\|\partial_{x}^{\alpha} \partial_{t} w_{\varepsilon \hat{\lambda}}(t)\right\|_{L^{2}}^{2}\right)^{1 / 2}\right] \\
& \leqq\left\{10 M+C_{1}(1+\eta)^{6} \eta\right\} \eta(1+t)^{-3}(\log (2+t))^{4} \\
& \times \sup _{t \geqq 0}\left[(1+t)^{3 / 2}(\log (2+t))^{-2}\left(\sum_{|\alpha| \leqq 1}\left\|\partial_{x}^{\alpha} \partial_{t} w_{\varepsilon \dot{\lambda}}(t)\right\|_{L^{2}}^{2}\right)^{1 / 2}\right], t \geqq 0,
\end{aligned}
$$

where $C_{1}$ does not depend on $\varepsilon$ and $\lambda$. If we choose $\eta>0$ so small that

$$
C_{1}(1+\eta)^{6} \eta \leqq M
$$


then we obtain by $(3.27)$

$$
\begin{gathered}
\sup _{t \geqq 0}\left[( 1 + t ) ^ { 3 / 2 } ( \operatorname { l o g } ( 2 + t ) ) ^ { - 2 } \left(\sum_{1 \leqq|\alpha| \leqq 2}\left\|\partial_{x}^{\alpha} w_{\varepsilon \lambda}(t)\right\|_{L^{2}}^{2}\right.\right. \\
\left.\left.+\sum_{|x| \leqq 1}\left\|\partial_{x}^{\alpha} \partial_{t} w_{\varepsilon \lambda}(t)\right\|_{L^{2}}^{2}\right)^{1 / 2}\right] \leqq 11 \eta M .
\end{gathered}
$$

In the same way as above, we have by (3.11), (3.18), (2.36) and Lemma 2.6

$$
\begin{aligned}
\sum_{|\alpha|=2} & \left\|\partial_{x}^{\alpha} \partial_{t} w_{\varepsilon \lambda}(t)\right\|_{L^{2}}^{2}+\sum_{|\alpha|=3}\left\|\partial_{x}^{\alpha} w_{\varepsilon \dot{\lambda}}(t)\right\|_{L^{2}}^{2} \\
\leqq & \left\{10 M+C_{2}(1+\eta)^{6} \eta\right\} \eta(1+t)^{-2}(\log (2+t))^{4} \\
\quad & \quad \sup _{t \geqq 0}\left[(1+t)(\log (2+t))^{-2}\left(\sum_{|\alpha|=2}\left\|\partial_{x}^{\alpha} \partial_{t} w_{\varepsilon \lambda}(t)\right\|_{L^{2}}^{2}\right)^{1 / 2}\right], \quad t \geqq 0,
\end{aligned}
$$

where $C_{2}$ does not depend on $\varepsilon$ and $\lambda$. If we choose $\eta>0$ so small that

$$
C_{2}(1+\eta)^{6} \eta \leqq M
$$

then we obtain by $(3.30)$

$$
\begin{gathered}
\sup _{t \geqq 0}\left[( 1 + t ) ( \operatorname { l o g } ( 2 + t ) ) ^ { - 2 } \left(\sum_{|x|=3}\left\|\partial_{x}^{\alpha} w_{\varepsilon \lambda}(t)\right\|_{L^{2}}^{2}\right.\right. \\
\left.\left.+\sum_{|x|=2}\left\|\partial_{x}^{\alpha} \partial_{t} w_{\varepsilon \lambda}(t)\right\|_{L^{2}}^{2}\right)^{1 / 2}\right] \leqq 11 M \eta .
\end{gathered}
$$

From (3.19), (3.8), (3.17), (3.18), (3.29) and (3.32), it follows that for some $C>0$,

$$
\begin{aligned}
& \sup _{t \geqq 0}\left[(1+t)^{3 / 2}(\log (2+t))^{-2}\left\|\partial_{t}^{2} w_{\varepsilon \dot{\lambda}}(t)\right\|_{L^{2}}\right. \\
& \left.\quad+(1+t)(\log (2+t))^{-2} \sum_{j+|\alpha|=1}\left\|\partial_{x}^{\alpha} \partial_{t}^{2+j} w_{\varepsilon \lambda}(t)\right\|_{L^{2}}\right] \leqq C,
\end{aligned}
$$

where $C$ does not depend on $\varepsilon$ and $\lambda$. When we consider passage to the limit as $\varepsilon \rightarrow+0$ and $\lambda \rightarrow+0,(3.29),(3.32),(3.33)$, a compactness argument and the regularity theorem of linear hyperbolic equation show the existence of the solution $w(t)$ to (3.13)-(3.14) such that

$$
\begin{gathered}
w(t) \in C\left([0, \infty) ; \dot{H}^{3} \cap \dot{H}^{1}\right), \\
\partial_{t} w(t) \in \bigcap_{j=0}^{2} C^{j}\left([0, \infty) ; H^{2-j}\right), \\
\sup _{t \geqq 0}\left[( 1 + t ) ^ { 3 / 2 } ( \operatorname { l o g } ( 2 + t ) ) ^ { - 2 } \left(\sum_{1 \leqq|\alpha| \leqq 2}\left\|\partial_{x}^{\alpha} w(t)\right\|_{L^{2}}^{2}\right.\right. \\
\left.\left.+\sum_{|\alpha| \leqq 1}\left\|\partial_{x}^{\alpha} \partial_{t} w(t)\right\|_{L^{2}}^{2}\right)^{1 / 2}\right] \leqq 11 M \eta,
\end{gathered}
$$




$$
\begin{gathered}
\sup _{t \geqq 0}\left[( 1 + t ) ( \operatorname { l o g } ( 2 + t ) ) ^ { - 2 } \left(\sum_{|\alpha|=3}\left\|\partial_{x}^{\alpha} w(t)\right\|_{L^{2}}^{2}\right.\right. \\
\left.\left.+\sum_{|\alpha|=2}\left\|\partial_{x}^{\alpha} \partial_{t} w(t)\right\|_{L^{2}}^{2}\right)^{1 / 2}\right] \leqq 11 M \eta \\
\sup _{t \geqq 0}\left[(1+t)^{3 / 2}(\log (2+t))^{-2}\left\|\partial_{t}^{2} w(t)\right\|_{L^{2}}\right. \\
\left.+(1+t)(\log (2+t))^{-2} \sum_{j+|\alpha|=1}\left\|\partial_{x}^{\alpha} \partial_{t}^{2+j} w(t)\right\|_{L^{2}}\right] \leqq C .
\end{gathered}
$$

The uniqueness of the solution follows from the same argument as in the proof of Proposition 2.3.

We next evaluate $z=N_{2}[v, u]$. By Lemmas 2.2(i), 2.4 and the GagliardoNirenberg inequality (see, e.g., [4]) we have

$$
\begin{aligned}
& \sum_{|\alpha| \leqq 2}\left\|\partial_{x}^{\alpha} G_{1}(t, v, u)\right\|_{L^{2}} \\
& \leqq C\left(\left\|A_{+}\right\|_{W^{3, x}}+\|R\|_{W^{3, \infty}}\right)\|u\|_{H^{2}} \\
& +C\left(\|\operatorname{div} v\|_{H^{2}}\|u\|_{L^{\infty}}+\|\operatorname{div} v\|_{W^{1,4}}\|u\|_{W^{1,4}}+\|\operatorname{div} v\|_{L^{x}}\|u\|_{H^{2}}\right) \\
& +C\left(1+\|\nabla \varphi\|_{W^{1, \infty}}\right)^{2}\left(\|v\|_{L^{6}}\left\|\frac{x}{t} m\right\|_{W^{2,3}}+\|v\|_{\dot{H}^{2} \cap \dot{H}^{1}}\left\|\frac{x}{t} m\right\|_{W^{1, \infty}}\right) \\
& +C\left(1+\|\nabla \varphi\|_{W^{1, \infty}}\right)^{2}\left(\|v\|_{L^{\infty}}\|\nabla \varphi\|_{W^{2, \infty}}\|m\|_{H^{2}}\right. \\
& \left.+\|v\|_{\dot{H}^{2} \cap \dot{H}^{1}}\|\nabla \varphi\|_{W^{1, \infty}}\|m\|_{H^{1}}\right) \\
& +C\left(1+\|\nabla \varphi\|_{W^{1, \infty}}\right)^{2} \frac{\theta(t)}{t}\left(\|v\|_{L^{\infty}}\left\|(i t)^{-3 / 2} e^{\frac{i|x|^{2}}{2 t}}\left(\nabla \hat{\psi}_{+0}\right)\left(\frac{x}{t}\right)\right\|_{H^{2}}\right. \\
& \left.+\|v\|_{\dot{H}^{2} \cap \dot{H}^{1}}\left\|(i t)^{-3 / 2} e^{\frac{i|x|^{2}}{2 t}}\left(\nabla \hat{\psi}_{+0}\right)\left(\frac{x}{t}\right)\right\|_{W^{1, x}}\right) \\
& +C\left(1+\|\nabla \varphi\|_{W^{1, \infty}}\right)^{2}\left\{\left(\left\|A_{+}\right\| W_{W^{2, \infty}}+\|R\|_{W^{2, \infty}}\right)\|v\|_{L^{\infty}}\|m\|_{H^{2}}\right. \\
& \left.+\left(\left\|A_{+}\right\|_{W^{1, \infty}}+\|R\|_{W^{1, \infty}}\right)\|v\|_{\dot{H}^{2} \cap \dot{H}^{1}}\|m\|_{L^{\infty}}\right\} \\
& +C\left(\left\|A_{+}\right\|_{W^{2, x}}+\|R\|_{W^{2, \infty}}\right)\|v\|_{L^{\alpha}}\|u\|_{H^{2}} \\
& +C\left(\left\|A_{+}\right\|_{W^{1, \infty}}+\|R\|_{W^{1, \infty}}\right)\left(\sum_{j=1}^{3}\left\|\nabla v_{j}\right\|_{L^{4}}\right)\|u\|_{W^{1,4}} \\
& +C\left(1+\|\nabla \varphi\|_{W^{2, \alpha}}\right)^{2}\left(\|v\|_{L^{\infty}}^{2}\|m\|_{H^{2}}+\|v\|_{L^{\infty}}\|v\|_{\dot{H}^{1}}\|m\|_{W^{1, \infty}}\right. \\
& \left.+\sum_{j=1}^{3}\left\|\nabla v_{j}\right\|_{L^{4}}^{2}\|m\|_{L^{\infty}}+\|v\|_{L^{\infty}}\|v\|_{\dot{H}^{2}}\|m\|_{L^{\infty}}\right)
\end{aligned}
$$




$$
\begin{gathered}
+C\left(\|v\|_{L^{\infty}}^{2}\|u\|_{H^{2}}+\|v\|_{L^{6}}\|v\|_{\dot{H}^{1}}\|u\|_{W^{1,3}}\right. \\
\left.+\sum_{j=1}^{3}\left\|\nabla v_{j}\right\|_{L^{4}}^{2}\|u\|_{L^{\infty}}+\|v\|_{L^{\infty}}\|v\|_{\dot{H}^{1}}\|u\|_{L^{\infty}}\right) \\
\leqq C(1+\eta)^{3} \eta^{2}(1+t)^{-2}(\log (2+t))^{2}, \quad t \geqq 0
\end{gathered}
$$

for $[v, u] \in X$. In the same way as above we have

$$
\sum_{|\alpha| \leqq 1}\left\|\partial_{x}^{\alpha} \partial_{t} G_{1}(t, v, u)\right\|_{L^{2}} \leqq C(1+\eta) \eta^{2}(1+t)^{-2}(\log (2+t))^{2}, \quad t \geqq 0
$$

for $[v, u] \in X$.

On the other hand, by (2.37), the Hardy-Littlewood-Sobolev inequality (see, e.g., [16]) and the Gagliardo-Nirenberg inequality (see, e.g., [4]) we have

$$
\begin{aligned}
& \sum_{|\alpha| \leqq 2}\left\|\partial_{x}^{\alpha} G_{2}(t, v, u)\right\|_{L^{2}} \leqq C\left\|\frac{1}{|x|} * \mathfrak{R}\left(e^{i \varphi} m \bar{u}\right)\right\|_{W^{2, \infty}}\left\|e^{\imath \varphi} m\right\|_{H^{2}} \\
& +C\left\|\frac{1}{|x|} *|m|^{2}\right\|_{W^{2, \infty}}\|u\|_{H^{2}}+C\left\|\frac{1}{|x|} *|u|^{2}\right\|_{W^{2,6}}\left\|e^{l \varphi} m\right\|_{W^{2,3}} \\
& +C\left\|\frac{1}{|x|} * \Re\left(e^{l \varphi} m \bar{u}\right)\right\|_{W^{2, \infty}}\|u\|_{H^{2}}+C\left\|\frac{1}{|x|} *|u|^{2}\right\|_{W^{2, \infty}}\|u\|_{H^{2}} \\
& \leqq C\left(1+\|\nabla \varphi\|_{W^{1, \infty}}\right)^{4}\|m \bar{u}\|_{H^{2}}^{1 / 2}\|m \bar{u}\|_{W^{2,6 / 5}}^{1 / 2}\|m\|_{H^{2}} \\
& +C\|m \bar{m}\|_{H^{2}}^{1 / 2}\|m \bar{m}\|_{W^{2,6 / 5}}^{1 / 2}\|u\|_{H^{2}} \\
& +C\left(1+\|\nabla \varphi\|_{W^{1, \infty}}\right)^{2}\|u \bar{u}\|_{W^{2,6 / 5}}^{1 / 2}\|m\|_{W^{2,3}} \\
& +C\left(1+\|\nabla \varphi\|_{W^{1, \infty}}\right)^{2}\|m \bar{u}\|_{H^{2}}^{1 / 2}\|m \bar{u}\|_{W^{2,6 / 5}}^{1 / 2}\|u\|_{H^{2}} \\
& +\|u \bar{u}\|_{H^{2}}^{1 / 2}\|u \bar{u}\|_{W^{2,6,5}}^{1 / 2}\|u\|_{H^{2}} \\
& \leqq C\left(1+\|\nabla \varphi\|_{W^{1, \infty}}\right)^{4}\|m\|_{W^{2, \infty}}^{1 / 2}\|u\|_{H^{2}}^{1 / 2}\|m\|_{W^{2,3}}^{1 / 2}\|u\|_{H^{2}}^{1 / 2}\|m\|_{H^{2}} \\
& +C\|m\|_{W^{2, \infty}}^{1 / 2}\|m\|_{H^{2}}^{1 / 2}\|m\|_{W^{2,3}}^{1 / 2}\|m\|_{H^{2}}^{1 / 2}\|u\|_{H^{2}} \\
& +C\left(1+\|\nabla \varphi\|_{W^{1, \infty}}\right)^{2}\left(\|u\|_{H^{2}}\|\bar{u}\|_{L^{3}}+\|u\|_{H^{1}}\|\bar{u}\|_{W^{1,3}}\right. \\
& \left.+\|u\|_{L^{3}}\|\bar{u}\|_{H^{2}}\right)\|m\|_{W^{2,3}} \\
& +C\left(1+\|\nabla \varphi\|_{W^{1, \infty}}\right)^{2}\|m\|_{W^{2, \infty}}^{1 / 2}\|u\|_{H^{2}}^{1 / 2}\|m\|_{W^{2,3}}^{1 / 2}\|u\|_{H^{2}}^{1 / 2}\|u\|_{H^{2}} \\
& +C\left(\|u\|_{H^{2}}\|\bar{u}\|_{L^{\infty}}+\|u\|_{W^{1,4}}\|\bar{u}\|_{W^{1,4}}+\|u\|_{L^{\infty}}\|\bar{u}\|_{H^{2}}\right)^{1 / 2} \\
& \times\left(\|u\|_{H^{2}}\|\bar{u}\|_{L^{3}}+\|u\|_{H^{1}}\|\bar{u}\|_{W^{1,3}}+\|u\|_{L^{3}}\|\bar{u}\|_{H^{2}}\right)^{1 / 2}\|u\|_{H^{2}} \\
& \leqq C(1+\eta)^{4} \eta^{3}(1+t)^{-2}(\log (2+t))^{2}, \quad t \geqq 0
\end{aligned}
$$

for $[v, u] \in X$. In the same way as above we have

$$
\sum_{|\alpha| \leqq 1}\left\|\partial_{x}^{\alpha} \partial_{t} G_{2}(t, v, u)\right\|_{L^{2}} \leqq C(1+\eta)^{4} \eta^{3}(1+t)^{-2}(\log (2+t))^{2}, \quad t \geqq 0
$$

for $[v, u] \in X$. 
We first consider the regularized problem associated with (3.15)-(3.16):

$$
\begin{gathered}
2 i \partial_{t} z_{\varepsilon \lambda}+\Delta z_{\varepsilon \lambda}=(1+\lambda t)^{-4}\left[2 i \rho_{\varepsilon} *\left\{\left(A_{+}+R+v\right) \cdot \nabla\left(\rho_{\varepsilon} * z_{\varepsilon \lambda}\right)\right\}\right. \\
\left.+\rho_{\varepsilon} * g(t)+\rho_{\varepsilon} * G_{1}(t, v, u)+\rho_{\varepsilon} * G_{2}(t, v, u)\right], \quad t \geqq 0, \quad x \in \mathbf{R}^{3}, \\
\left\|z_{\varepsilon \lambda}(t)\right\|_{L^{2}} \rightarrow 0 \quad(t \rightarrow \infty)
\end{gathered}
$$

where $0<\varepsilon \leqq 1$ and $0<\lambda<1 / 4$. By using the contraction mapping principle, we easily see that for each $\varepsilon$ and $\lambda$, there exists a $T>0$ such that (3.43)-(3.44) has the unique solution $z_{\varepsilon \lambda}(t)$ satisfying

$$
\begin{gathered}
z_{\varepsilon \lambda}(t) \in \bigcap_{j=1}^{\infty} C^{2}\left([T, \infty) ; H^{j}\right), \\
\sup _{t \geqq T}\left[(1+\lambda t)^{4} \sum_{j=0}^{2}\left\|\partial_{x}^{\alpha} \partial_{t}^{j} z_{\varepsilon \hat{\lambda}}(t)\right\|_{L^{2}}\right]<+\infty .
\end{gathered}
$$

Since the initial value problem of (3.43) can easily be solved globally in time, the above solution $z_{\varepsilon \lambda}(t)$ can be extended to the time interval $[0, \infty)$.

We next derive the a priori estimates independent of $\varepsilon$ and $\lambda$ for $z_{\varepsilon \lambda}$. Let $\alpha$ be an arbitrary multi-index with $|\alpha| \leqq 2$. We apply $\partial_{x}^{\alpha}$ to (3.43) and take the scalar product in $L^{2}$ between the resulting equation and $\partial_{x}^{\alpha} z_{\varepsilon \lambda}(t)$ to obtain

$$
\begin{aligned}
& i \frac{d}{d t}\left\|\partial_{x}^{\alpha} z_{\varepsilon \lambda}(t)\right\|_{L^{2}}^{2}=\left\|\nabla \partial_{x}^{\alpha} z_{\varepsilon \lambda}(t)\right\|_{L^{2}}^{2} \\
& \quad+2 i(1+\lambda t)^{-4}\left(\rho_{\varepsilon} *\left\{\left(A_{+}+R+v\right) \cdot \nabla \partial_{x}^{\alpha}\left(\rho_{\varepsilon} * z_{\varepsilon \lambda}\right)\right\}, \partial_{x}^{\alpha} z_{\varepsilon \lambda}\right) \\
& \quad+(1+\lambda t)^{-4} \sum_{\substack{\alpha_{1}+\alpha_{2}=\alpha \\
\left|\alpha_{2}\right| \leqq|\alpha|-1}} C_{\alpha_{1} \alpha_{2}}\left(2 i \rho_{\varepsilon} *\left\{\partial_{x}^{\alpha_{1}}\left(A_{+}+R+v\right) \cdot \nabla \partial_{x}^{\alpha_{2}}\left(\rho_{\varepsilon} * z_{\varepsilon \lambda}\right)\right\}, \partial_{x}^{\alpha} z_{\varepsilon \lambda}\right) \\
& \quad+(1+\lambda t)^{-4}\left(\rho_{\varepsilon} *\left(\partial_{x}^{\alpha} g+\partial_{x}^{\alpha} G_{1}(t, v, u)+\partial_{x}^{\alpha} G_{2}(t, v, u)\right), \partial_{x}^{\alpha} z_{\varepsilon \lambda}\right), \quad t \geqq 0
\end{aligned}
$$

Here, the summation at the right-hand side of (3.47) is empty, when $|\alpha|=0$. By the integration by parts we have

$$
\begin{aligned}
& \left(\rho_{\varepsilon} *\left\{\left(A_{+}+R+v\right) \cdot \nabla \partial_{x}^{\alpha}\left(\rho_{\varepsilon} * z_{\varepsilon \lambda}\right)\right\}, \partial_{x}^{\alpha} z_{\varepsilon \lambda}\right) \\
= & \left(\left(A_{+}+R+v\right) \cdot \nabla\left(\rho_{\varepsilon} * \partial_{x}^{\alpha} z_{\varepsilon \lambda}\right), \rho_{\varepsilon} * \partial_{x}^{\alpha} z_{\varepsilon \lambda}\right) \\
= & -\frac{1}{2}\left(\operatorname{div}\left(A_{+}+R+v\right)\left(\rho_{\varepsilon} * \partial_{x}^{\alpha} z_{\varepsilon \lambda}\right), \rho_{\varepsilon} * \partial_{x}^{\alpha} z_{\varepsilon \lambda}\right) .
\end{aligned}
$$

We take the imaginary part of (3.47) to obtain by (3.48)

$$
\begin{aligned}
\frac{d}{d t}\left\|\partial_{x}^{\alpha} z_{\varepsilon \lambda}(t)\right\|_{L^{2}}^{2} & \\
= & -(1+\lambda t)^{-4}\left(\operatorname{div}\left(A_{+}+R+v\right)\left(\rho_{\varepsilon} * \partial_{x}^{\alpha} z_{\varepsilon \lambda}\right), \rho_{\varepsilon} * \partial_{x}^{\alpha} z_{\varepsilon \lambda}\right) \\
& +2(1+\lambda t)^{-4} \sum_{\substack{\alpha_{1}+\alpha_{2}=x \\
\left|\alpha_{2}\right| \leqq|\alpha|-1}} C_{\alpha_{1} \alpha_{2}} \mathfrak{R}\left(\rho_{\varepsilon} *\left\{\partial_{x}^{\alpha_{1}}\left(A_{+}+R+v\right) \cdot \nabla \partial_{x}^{\alpha_{2}}\left(\rho_{\varepsilon} * z_{\varepsilon \lambda}\right)\right\}, \partial_{x}^{\alpha} z_{\varepsilon \lambda}\right) \\
& +(1+\lambda t)^{-4} \mathfrak{J}\left(\rho_{\varepsilon} *\left(\partial_{x}^{\alpha} g+\partial_{x}^{\alpha} G_{1}+\partial_{x}^{\alpha} G_{2}\right), \partial_{x}^{\alpha} z_{\varepsilon \lambda}\right)
\end{aligned}
$$


$\leqq\left(\left\|\operatorname{div} A_{+}\right\|_{L^{\infty}}+\|\operatorname{div} R\|_{L^{\infty}}+\|\operatorname{div} v\|_{L^{\infty}}\right)\left\|\partial_{x}^{\alpha} z_{\varepsilon \lambda}\right\|_{L^{2}}^{2}$

$+C\left(\left\|A_{+}\right\|_{W|x|, \infty}+\|R\|_{W|x|, \infty}\right)\left\|z_{\varepsilon \lambda}\right\|_{H^{|x|}}^{2}$

$+C\left\|\partial_{x}^{\alpha} v\right\|_{L^{3}}\left\|\nabla z_{\varepsilon \lambda}\right\|_{L^{6}}^{2}\left\|\partial_{x}^{\alpha} z_{\varepsilon \lambda}\right\|_{L^{2}}+C\|v\|_{W^{|\alpha|-1, \infty}}\left\|z_{\varepsilon \lambda}\right\|_{H^{|\gamma|}}^{2}$

$+\left(\left\|\partial_{x}^{\alpha} g\right\|_{L^{2}}+\left\|\partial_{x}^{\alpha} G_{1}\right\|_{L^{2}}+\left\|\partial_{x}^{\alpha} G_{2}\right\|_{L^{2}}\right)\left\|\partial_{x}^{\alpha} z_{\varepsilon \lambda}\right\|_{L^{2}}, \quad t \geqq 0$,

if $1 \leqq|\alpha| \leqq 2$. We take the summation of (3.49) with respect to $\alpha$ and use (3.12), (3.39), (3.41), the Gagliardo-Nirenberg inequality and Lemmas 2.2(i), 2.4 to obtain

$$
\begin{aligned}
& \frac{d}{d t}\left(\sum_{1 \leqq|\alpha| \leqq 2}\left\|\partial_{x}^{\alpha} z_{\varepsilon \lambda}(t)\right\|_{L^{2}}^{2}\right) \\
& \leqq C(1+\eta) \eta(1+t)^{-1} \sum_{|\alpha| \leqq 2}\left\|\partial_{x}^{\alpha} z_{\varepsilon \lambda}(t)\right\|_{L^{2}}^{2} \\
&+C \eta^{2}(1+t)^{-5 / 4}(\log (2+t))^{2} \sum_{|\alpha| \leqq 2}\left\|\partial_{x}^{\alpha} z_{\varepsilon \lambda}(t)\right\|_{L^{2}}^{2} \\
&+C(1+\eta) \eta(1+t)^{-1} \sum_{|\alpha| \leqq 2}\left\|\partial_{x}^{\alpha} z_{\varepsilon \lambda}(t)\right\|_{L^{2}}^{2} \\
&+C \eta(1+t)^{-5 / 4}(\log (2+t))^{2} \sum_{|\alpha| \leqq 2}\left\|\partial_{x}^{\alpha} z_{\varepsilon \lambda}(t)\right\|_{L^{2}}^{2} \\
&+\sum_{1 \leqq|\alpha| \leqq 2}\left\|\partial_{x}^{\alpha} g\right\|_{L^{2}}\left\|\partial_{x}^{\alpha} z_{\varepsilon \lambda}(t)\right\|_{L^{2}}^{2} \\
&+C(1+\eta)^{3} \eta^{2}(1+t)^{-2}(\log (2+t))^{2} \sum_{|\alpha| \leqq 2}\left\|\partial_{x}^{\alpha} z_{\varepsilon \lambda}(t)\right\|_{L^{2}}^{2} \\
&+C(1+\eta)^{4} \eta^{3}(1+t)^{-2}(\log (2+t))^{2} \sum_{|\alpha| \leqq 2}\left\|\partial_{x}^{\alpha} z_{\varepsilon \lambda}(t)\right\|_{L^{2}}^{2}, \quad t \geqq 0 .
\end{aligned}
$$

If $|\alpha|=0$, we have by (3.47), (3.48) with $|\alpha|=0$ and Lemmas 2.2(i), 2.4

$$
\begin{aligned}
\frac{d}{d t}\left\|z_{\varepsilon \lambda}(t)\right\|_{L^{2}}^{2}= & -(1+\lambda t)^{-4}\left(\operatorname{div}\left(A_{+}+R+v\right)\left(\rho_{\varepsilon} * z_{\varepsilon \dot{\lambda}}\right), z_{\varepsilon \lambda}\right) \\
& +(1+\lambda t)^{-4} \mathfrak{J}\left(\rho_{\varepsilon} *\left(g+G_{1}+G_{2}\right), z_{\varepsilon \lambda}\right) \\
\leqq & C(1+\eta) \eta(1+t)^{-1}\left\|z_{\varepsilon \lambda}(t)\right\|_{L^{2}}^{2}+C\|v\|_{\dot{H}^{2}}^{1 / 2}\|v\|_{\dot{H}^{1}}^{1 / 2}\left\|z_{\varepsilon \lambda}(t)\right\|_{L^{2}}^{2} \\
& +\left(\|g\|_{L^{2}}+\left\|G_{1}\right\|_{L^{2}}+\left\|G_{2}\right\|_{L^{2}}\right)\left\|z_{\varepsilon \lambda}(t)\right\|_{L^{2}} \\
\leqq & C(1+\eta) \eta(1+t)^{-1}\left\|z_{\varepsilon \lambda}(t)\right\|_{L^{2}}^{2} \\
& +C \eta(1+t)^{-3 / 2}(\log (2+t))^{2}\left\|z_{\varepsilon \hat{\lambda}}(t)\right\|_{L^{2}}^{2} \\
& +\left(\|g\|_{L^{2}}+\left\|G_{1}\right\|_{L^{2}}+\left\|G_{2}\right\|_{L^{2}}\right)\left\|z_{\varepsilon \lambda}(t)\right\|_{L^{2}}, \quad t \geqq 0
\end{aligned}
$$

Equations (3.50), (3.51), (3.12), the Hölder inequality for sequence and Lemma 2.6 yield

$$
\begin{aligned}
\sum_{|\alpha| \leqq 2}\left\|\partial_{x}^{\alpha} z_{\varepsilon \lambda}(t)\right\|_{L^{2}}^{2} \leqq & C(1+\eta)^{6} \eta \int_{t}^{\infty}(1+s)^{-1} \sum_{|\alpha| \leqq 2}\left\|\partial_{x}^{\alpha} z_{\varepsilon \lambda}(s)\right\|_{L^{2}}^{2} d s \\
& +\int_{t}^{\infty} \sum_{|\alpha| \leqq 2}\left\|\partial_{x}^{\alpha} g(s)\right\|_{L^{2}}\left\|\partial_{x}^{\alpha} z_{\varepsilon \lambda}(s)\right\|_{L^{2}} d s
\end{aligned}
$$




$$
\begin{aligned}
\leqq & C(1+\eta)^{6} \eta \int_{t}^{\infty}(1+s)^{-3}(\log (2+s))^{4} d s \\
& \times\left\{\sup _{t \geqq 0}\left[(1+t)(\log (2+t))^{-2}\left(\sum_{|\alpha| \leqq 2}\left\|\partial_{x}^{\alpha} z_{\varepsilon \lambda}(t)\right\|_{L^{2}}^{2}\right)^{1 / 2}\right]\right\}^{2} \\
& +\int_{t}^{\infty}\left(\sum_{|\alpha| \leqq 2}\left\|\partial_{x}^{\alpha} g(s)\right\|_{L^{2}}^{2}\right)^{1 / 2}\left(\sum_{|\alpha| \leqq 2}\left\|\partial_{x}^{\alpha} z_{\varepsilon \lambda}(s)\right\|_{L^{2}}^{2}\right)^{1 / 2} d s \\
\leqq & C_{3}(1+\eta)^{6} \eta(1+t)^{-2}(\log (2+t))^{4} \\
& \times\left\{\sup _{t \geqq 0}\left[(1+t)(\log (2+t))^{-2}\left(\sum_{|\alpha| \leqq 2}\left\|\partial_{x}^{\alpha} z_{\varepsilon \lambda}(t)\right\|_{L^{2}}^{2}\right)^{1 / 2}\right]\right\}^{2} \\
& +5 M \eta(1+t)^{-2}(\log (2+t))^{4} \\
& \times \sup _{t \geqq 0}\left[(1+t)(\log (2+t))^{-2}\left(\sum_{|\alpha| \leqq 2}\left\|\partial_{x}^{\alpha} z_{\varepsilon \lambda}(t)\right\|_{L^{2}}^{2}\right)^{1 / 2}\right], t \geqq 0,
\end{aligned}
$$

where $C_{3}$ does not depend on $\varepsilon$ and $\lambda$. If we choose $\eta>0$ so small that

$$
C_{3}(1+\eta)^{6} \eta \leqq \frac{1}{2}
$$

then we have by (3.52)

$$
\sup _{t \geqq 0}\left[(1+t)(\log (2+t))^{-2}\left(\sum_{|\alpha| \leqq 2}\left\|\partial_{x}^{\alpha} z_{\varepsilon \lambda}(t)\right\|_{L^{2}}^{2}\right)^{1 / 2}\right] \leqq 6 M \eta .
$$

Differentiating (3.43) in $t$, we have

$$
\begin{aligned}
2 i \partial_{t}\left(\partial_{t} z_{\varepsilon \lambda}\right)+\Delta\left(\partial_{t} z_{\varepsilon \lambda}\right)= & 2 i(1+\lambda t)^{-4} \rho_{\varepsilon} *\left\{\left(A_{+}+R+v\right) \cdot \nabla\left(\rho_{\varepsilon} * \partial_{t} z_{\varepsilon \lambda}\right)\right\} \\
& -8 i \lambda(1+\lambda t)^{-5} \rho_{\varepsilon} *\left\{\left(A_{+}+R+v\right) \cdot \nabla\left(\rho_{\varepsilon} * \partial_{t} z_{\varepsilon \lambda}\right)\right\} \\
& +2 i(1+\lambda t)^{-4} \rho_{\varepsilon} *\left\{\left(\partial_{t} A_{+}+\partial_{t} R+\partial_{t} v\right) \cdot \nabla\left(\rho_{\varepsilon} * z_{\varepsilon \lambda}\right)\right\} \\
& +\partial_{t}\left[(1+\lambda t)^{-4} \rho_{\varepsilon} *\left\{g+G_{1}+G_{2}\right\}\right], \quad t \geqq 0 .
\end{aligned}
$$

We take the scalar product in $L^{2}$ between (3.55) and $\partial_{t} z_{\varepsilon \lambda}(t)$ and take the imaginary part of the resulting equality to obtain by (3.48), (3.54), (3.42), Lemmas 2.2(i), 2.4 and the Gagliardo-Nirenberg inequality

$$
\begin{aligned}
\frac{d}{d t}\left\|\partial_{t} z_{\varepsilon \lambda}(t)\right\|_{L^{2}}^{2} \leqq & \left(\left\|\operatorname{div} A_{+}\right\|_{L^{\infty}}+\|\operatorname{div} R\|_{L^{\infty}}+\|\operatorname{div} v\|_{L^{\infty}}\right)\left\|\partial_{t} z_{\varepsilon \lambda}\right\|_{L^{2}}^{2} \\
& +C\left(\left\|A_{+}\right\|_{L^{\infty}}+\|R\|_{L^{\infty}}+\|v\|_{L^{\infty}}\right)\left\|\nabla z_{\varepsilon \lambda}\right\|_{L^{2}}\left\|\partial_{t} z_{\varepsilon \lambda}\right\|_{L^{2}} \\
& +C\left(\left\|\partial_{t} A_{+}\right\|_{L^{\infty}}+\left\|\partial_{t} R\right\|_{L^{\infty}}+\left\|\partial_{t} v\right\|_{L^{\infty}}\right)\left\|\nabla z_{\varepsilon \lambda}\right\|_{L^{2}}\left\|\partial_{t} z_{\varepsilon \lambda}\right\|_{L^{2}} \\
& +\left(\|g\|_{L^{2}}+\left\|G_{1}\right\|_{L^{2}}+\left\|G_{2}\right\|_{L^{2}}\right)\left\|\partial_{t} z_{\varepsilon \lambda}\right\|_{L^{2}} \\
& +\left(\left\|\partial_{t} g\right\|_{L^{2}}+\left\|\partial_{t} G_{1}\right\|_{L^{2}}+\left\|\partial_{t} G_{2}\right\|_{L^{2}}\right)\left\|\partial_{t} z_{\varepsilon \lambda}\right\|_{L^{2}}
\end{aligned}
$$




$$
\begin{aligned}
\leqq & C(1+\eta) \eta(1+t)^{-1}\left\|\partial_{t} z_{\varepsilon \lambda}\right\|_{L^{2}}^{2} \\
& +C(1+\eta)^{5} \eta^{2}(1+t)^{-2}(\log (2+t))^{2}\left\|\partial_{t} z_{\varepsilon \lambda}\right\|_{L^{2}} \\
& +\left(\sum_{j=0}^{1}\left\|\partial_{t}^{j} g\right\|_{L^{2}}\right)\left\|\partial_{t} z_{\varepsilon \lambda}\right\|_{L^{2}}, \quad t \geqq 0 .
\end{aligned}
$$

Here, we have used the fact that $0<\lambda<\frac{1}{4}$ at the first inequality of (3.56). Let $\alpha$ be an arbitrary multi-index with $|\alpha|=1$. The application of $\partial_{x}^{\alpha}$ to (3.55) yields

$$
\begin{aligned}
2 i \partial_{t}\left(\partial_{x}^{\alpha} z_{\varepsilon \lambda}\right)+\Delta\left(\partial_{x}^{\alpha} z_{\varepsilon \lambda}\right)= & 2 i(1+\lambda t)^{-4} \rho_{\varepsilon} *\left\{\left(A_{+}+R+v\right) \cdot \nabla\left(\rho_{\varepsilon} * \partial_{x}^{\alpha} \partial_{t} z_{\varepsilon \lambda}\right)\right\} \\
& +2 i(1+\lambda t)^{-4} \rho_{\varepsilon} *\left\{\left(\partial_{x}^{\alpha} A_{+}+\partial_{x}^{\alpha} R+\partial_{x}^{\alpha} v\right) \cdot \nabla\left(\rho_{\varepsilon} * \partial_{t} z_{\varepsilon \lambda}\right)\right\} \\
& -8 i \lambda(1+\lambda t)^{-5} \rho_{\varepsilon} *\left\{\left(\partial_{x}^{\alpha} A_{+}+\partial_{x}^{\alpha} R+\partial_{x}^{\alpha} v\right) \cdot \nabla\left(\rho_{\varepsilon} * z_{\varepsilon \lambda}\right)\right. \\
& \left.+\left(A_{+}+R+v\right) \cdot \nabla\left(\rho_{\varepsilon} * \partial_{x}^{\alpha} z_{\varepsilon \lambda}\right)\right\} \\
& +2 i(1+\lambda t)^{-4} \rho_{\varepsilon} *\left\{\left(\partial_{x}^{\alpha} \partial_{t} A_{+}+\partial_{x}^{\alpha} \partial_{t} R+\partial_{x}^{\alpha} \partial_{t} v\right) \cdot \nabla\left(\rho_{\varepsilon} * z_{\varepsilon \lambda}\right)\right. \\
& \left.+\left(\partial_{t} A_{+}+\partial_{t} R+\partial_{t} v\right) \cdot \nabla\left(\rho_{\varepsilon} * \partial_{x}^{\alpha} z_{\varepsilon \lambda}\right)\right\} \\
& +\partial_{x}^{\alpha} \partial_{t}\left[(1+\lambda t)^{-4} \rho_{\varepsilon} *\left\{g+G_{1}+G_{2}\right\}\right], \quad t \geqq 0 .
\end{aligned}
$$

We take the scalar product in $L^{2}$ between (3.57) and $\partial_{x}^{\alpha} \partial_{t} z_{\varepsilon \lambda}(t)$ and take the imaginary part of the resulting equality to obtain by (3.48), (3.54), (3.42), Lemmas 2.2(i), 2.4 and the Gagliardo-Nirenberg inequality

$$
\begin{aligned}
& \frac{d}{d t}\left\|\partial_{x}^{\alpha} z_{\varepsilon \lambda}(t)\right\|_{L^{2}}^{2} \leqq\left(\left\|\operatorname{div} A_{+}\right\| L_{L^{\infty}}+\|\operatorname{div} R\|_{L^{\infty}}+\|\operatorname{div} v\|_{L^{\infty}}\right)\left\|\partial_{x}^{\alpha} \partial_{t} z_{\varepsilon \lambda}\right\|_{L^{2}}^{2} \\
& +C\left(\left\|\partial_{x}^{\alpha} A_{+}\right\|_{L^{\infty}}+\left\|\partial_{x}^{\alpha} R\right\|_{L^{\infty}}+\left\|\partial_{x}^{\alpha} v\right\|_{L^{\infty}}\right)\left\|\nabla \partial_{t} z_{\varepsilon \lambda}\right\|_{L^{2}}\left\|\partial_{x}^{\alpha} \partial_{t} z_{\varepsilon \lambda}\right\|_{L^{2}} \\
& +C\left(\left\|\partial_{x}^{\alpha} A_{+}\right\|_{L^{\infty}}+\left\|\partial_{x}^{\alpha} R\right\|_{L^{\infty}}+\left\|\partial_{x}^{\alpha} v\right\|_{L^{\infty}}\right)\left\|\nabla z_{\varepsilon \lambda}\right\|_{L^{2}}\left\|\partial_{x}^{\alpha} \partial_{t} z_{\varepsilon \lambda}\right\|_{L^{2}} \\
& +C\left(\left\|A_{+}\right\|_{L^{\infty}}+\|R\|_{L^{\infty}}+\|v\|_{L^{\infty}}\right)\left\|\nabla \partial_{x}^{\alpha} z_{\varepsilon \lambda}\right\|_{L^{2}}\left\|\partial_{x}^{\alpha} \partial_{t} z_{\varepsilon \lambda}\right\|_{L^{2}} \\
& +C\left(\left\|\partial_{x}^{\alpha} \partial_{t} A_{+}\right\|_{L^{\infty}}+\left\|\partial_{x}^{\alpha} \hat{o}_{t} R\right\|_{L^{\infty}}\right)\left\|\nabla z_{\varepsilon \lambda}\right\|_{L^{2}}\left\|\partial_{x}^{\alpha} \partial_{t} z_{\varepsilon \lambda}\right\|_{L^{2}} \\
& +C\left\|\partial_{x}^{\alpha} \partial_{t} v\right\|_{L^{4}}\left\|\nabla z_{\varepsilon \lambda}\right\|_{L^{4}}\left\|\partial_{x}^{\alpha} \partial_{t} z_{\varepsilon \lambda}\right\|_{L^{2}} \\
& +C\left(\left\|\partial_{t} A_{+}\right\|_{L^{\infty}}+\left\|\partial_{t} R\right\|_{L^{\infty}}+\left\|\partial_{t} v\right\|_{L^{\infty}}\right)\left\|\nabla \partial_{x}^{\alpha} z_{\varepsilon \lambda}\right\|_{L^{2}}\left\|\partial_{x}^{\alpha} \partial_{t} z_{\varepsilon \lambda}\right\|_{L^{2}} \\
& +\left(\left\|\partial_{x}^{\alpha} g\right\|_{L^{2}}+\left\|\partial_{x}^{\alpha} G_{1}\right\|_{L^{2}}+\left\|\partial_{x}^{\alpha} G_{2}\right\|_{L^{2}}\right)\left\|\partial_{x}^{\alpha} \partial_{t} z_{\varepsilon \lambda}\right\|_{L^{2}} \\
& +\left(\left\|\partial_{x}^{\alpha} \partial_{t} g\right\|_{L^{2}}+\left\|\partial_{x}^{\alpha} \partial_{t} G_{1}\right\|_{L^{2}}+\left\|\partial_{x}^{\alpha} \partial_{t} G_{2}\right\|_{L^{2}}\right)\left\|\partial_{x}^{\alpha} \partial_{t} z_{\varepsilon \lambda}\right\|_{L^{2}} \\
& \leqq C(1+\eta) \eta(1+t)^{-1} \sum_{|\beta|=1}\left\|\partial_{x}^{\beta} \partial_{t} z_{\varepsilon \lambda}\right\|_{L^{2}}^{2} \\
& +C(1+\eta) \eta^{2}(1+t)^{-2}(\log (2+t))^{2} \sum_{|\beta|=1}\left\|\partial_{x}^{\beta} \partial_{t} z_{\varepsilon \lambda}\right\|_{L^{2}} \\
& +C\left(1+\eta^{5}\right) \eta^{2}(1+t)^{-2}(\log (2+t))^{2}\left\|\partial_{x}^{\alpha} \partial_{t} z_{\varepsilon \lambda}\right\|_{L^{2}} \\
& +\left\{\sum_{j=0}^{1}\left\|\partial_{x}^{\alpha} \partial_{t}^{j} g\right\|_{L^{2}}\right\}\left\|\partial_{x}^{\alpha} \partial_{t} z_{\varepsilon \lambda}\right\|_{L^{2}}, \quad t \geqq 0 .
\end{aligned}
$$


Here, we have used the fact that $0<\lambda<1 / 4$ at the first inequality of (3.58). Equations (3.56), (3.58), (3.12), the Hölder inequality for sequence and Lemma 2.6 yield

$$
\begin{aligned}
& \sum_{|\alpha| \leqq 1}\left\|\partial_{x}^{\alpha} z_{\varepsilon \lambda}(t)\right\|_{L^{2}}^{2} \leqq C(1+\eta) \eta \int_{t}^{\infty}(1+s)^{-1} \sum_{|\alpha| \leqq 1}\left\|\partial_{x}^{\alpha} z_{\varepsilon \lambda}(s)\right\|_{L^{2}}^{2} d s \\
& +C(1+\eta)^{5} \eta^{2} \int_{t}^{\infty}(1+s)^{-2}(\log (2+s))^{2} \sum_{|\alpha| \leqq 1}\left\|\partial_{x}^{\alpha} z_{\varepsilon \lambda}(s)\right\|_{L^{2}} d s \\
& +\int_{t}^{\infty}\left\{\sum_{j=0}^{1}\left\|\partial_{t}^{j} g(s)\right\|_{L^{2}}\right\}\left\|\partial_{t} z_{\varepsilon \lambda}(s)\right\|_{L^{2}} d s \\
& +\int_{t}^{\infty}\left[\sum_{|\alpha| \leqq 1}\left\|\partial_{x}^{\alpha} \partial_{s} g(s)\right\|_{L^{2}}\left\|\partial_{x}^{\alpha} \partial_{s} z_{\varepsilon \lambda}(s)\right\|_{L^{2}}\right. \\
& \left.+\sum_{|\alpha| \leqq 1}\left\|\partial_{x}^{\alpha} g(s)\right\|_{L^{2}}\left\|\partial_{x}^{\alpha} \partial_{s} z_{\varepsilon \lambda}(s)\right\|_{L^{2}}\right] d s \\
& \leqq C_{4}(1+\eta) \eta(1+t)^{-2}(\log (2+t))^{4} \\
& \times\left\{\sup _{t \geqq 0}\left[(1+t)(\log (2+t))^{-2}\left(\sum_{|\alpha| \leqq 1}\left\|\partial_{x}^{\alpha} \partial_{t} z_{\varepsilon \lambda}(t)\right\|_{L^{2}}^{2}\right)^{1 / 2}\right]\right\}^{2} \\
& +C_{5}(1+\eta)^{5} \eta^{2}(1+t)^{-2}(\log (2+t))^{2} \\
& \times \sup _{t \geqq 0}\left[(1+t)(\log (2+t))^{-2}\left(\sum_{|\alpha| \leqq 1}\left\|\partial_{x}^{\alpha} \partial_{t} z_{\varepsilon \lambda}(t)\right\|_{L^{2}}^{2}\right)^{1 / 2}\right] \\
& +10 M \eta(1+t)^{-2}(\log (2+t))^{4} \\
& \times \sup _{t \geqq 0}\left[(1+t)(\log (2+t))^{-2}\right. \\
& \left.\times\left(\sum_{|\alpha| \leqq 1}\left\|\partial_{x}^{\alpha} \partial_{t} z_{\varepsilon \lambda}(t)\right\|_{L^{2}}^{2}\right)^{1 / 2}\right], \quad t \geqq 0,
\end{aligned}
$$

where $C_{4}$ and $C_{5}$ do not depend on $\varepsilon$ and $\lambda$. If we choose $\eta>0$ so small that

$$
\begin{gathered}
C_{4}(1+\eta) \eta \leqq \frac{1}{2}, \\
C_{5}(1+\eta)^{5} \eta \leqq \frac{1}{2} M,
\end{gathered}
$$

then we obtain by (3.58)

$$
\sup _{t \geqq 0}\left[(1+t)(\log (2+t))^{-2}\left(\sum_{|\alpha| \leqq 1}\left\|\partial_{x}^{\alpha} \partial_{t} z_{\varepsilon \lambda}(t)\right\|_{L^{2}}^{2}\right)^{1 / 2}\right] \leqq 21 M \eta
$$


Furthermore, from (3.43), (3.54), (3.62), (3.39), (3.41), the Hölder inequality for sequence and the Gagliardo-Nirenberg inequality it follows that

$$
\begin{aligned}
\sum_{|\alpha|=1}\left\|\Delta \partial_{x}^{\alpha} z_{\varepsilon \lambda}(t)\right\|_{L^{2}} \leqq & 2 \sum_{|\alpha|=1}\left\|\partial_{x}^{\alpha} \partial_{t} z_{\varepsilon \lambda}(t)\right\|_{L^{2}} \\
& +2 \sum_{|\alpha|=1}\left\|\partial_{x}^{\alpha} \rho_{\varepsilon} *\left\{\left(A_{+}+R+v\right) \cdot \nabla\left(\rho_{\varepsilon} * z_{\varepsilon \dot{\lambda}}\right)\right\}\right\|_{L^{2}} \\
& +\sum_{|\alpha|=1}\left\|\partial_{x}^{\alpha} \rho_{\varepsilon} *\left\{g+G_{1}+G_{2}\right\}\right\|_{L^{2}} \\
\leqq & 2 \sqrt{3}\left(\sum_{|\alpha|=1}\left\|\partial_{x}^{\alpha} \partial_{t} z_{\varepsilon \dot{\lambda}}(t)\right\|_{L^{2}}^{2}\right)^{1 / 2} \\
& +C\left(\left\|A_{+}\right\| W^{1, x}+\|R\|_{W^{1, \infty}}+\|v\|_{W^{1, \infty}}\right)\left\|z_{\varepsilon \dot{\lambda}}\right\|_{H^{2}} \\
& +\sum_{|\alpha|=1}\left(\left\|\partial_{x}^{\alpha} g\right\|_{L^{2}}+\left\|\partial_{x}^{\alpha} G_{1}\right\|_{L^{2}}+\left\|\partial_{x}^{\alpha} G_{2}\right\|_{L^{2}}\right) \\
\leqq & {\left[(42 \sqrt{3}+1) M \eta+C_{6}(1+\eta) \eta(M \eta)\right.} \\
& \left.+C_{7}\left\{1+(1+\eta)^{5}\right\} \eta^{2}\right](1+t)^{-1}(\log (2+t))^{2}, \quad t \geqq 0,
\end{aligned}
$$

where $C_{6}$ and $C_{7}$ do not depend on $\varepsilon$ and $\lambda$. If we choose $\eta>0$ so small that

$$
\begin{aligned}
C_{6}(1+\eta) \eta & \leqq 1, \\
C_{7}\left\{1+(1+\eta)^{5}\right\} \eta & \leqq M,
\end{aligned}
$$

then we obtain by (3.62),

$$
\sup _{t \geqq 0}\left[(1+t)(\log (2+t))^{-2} \sum_{|\alpha|=1}\left\|\Delta \partial_{x}^{\alpha} z_{\varepsilon \lambda}(t)\right\|_{L^{2}}\right] \leqq 3(14 \sqrt{3}+1) M \eta .
$$

Let $\alpha$ be an arbitrary multi-index with $|\alpha| \leqq 2$. The application of $\partial_{x}^{\alpha}$ to (3.43) yields

$$
\begin{aligned}
2 i \partial_{t}\left(\partial_{x}^{\alpha} z_{\varepsilon \lambda}\right)+\Delta\left(\partial_{x}^{\alpha} z_{\varepsilon \lambda}\right)= & (1+\lambda t)^{-4} \partial_{x}^{\alpha}\left[2 i \rho_{\varepsilon} *\left\{\left(A_{+}+R+v\right) \cdot \nabla\left(\rho_{\varepsilon} * z_{\varepsilon \lambda}\right)\right\}\right. \\
& \left.+\rho_{\varepsilon} * g+\rho_{\varepsilon} * G_{1}+\rho_{\varepsilon} * G_{2}\right], \quad t \geqq 0, \quad x \in \mathbf{R}^{3} .
\end{aligned}
$$

Since $z_{\varepsilon \lambda}(t) \rightarrow 0$ in $H^{2}$ as $t \rightarrow \infty$, we have by (3.67) and Duhamel's principle

$$
\begin{aligned}
\partial_{x}^{\alpha} z_{\varepsilon \lambda}(t)= & \frac{i}{2} \int_{t}^{\infty}(1+\lambda s)^{-4} U(t-s)\left[2 i \rho_{\varepsilon} * \partial_{x}^{\alpha}\left\{\left(A_{+}+R+v\right) \cdot \nabla\left(\rho_{\varepsilon} * z_{\varepsilon \lambda}\right)\right\}\right. \\
& \left.+\rho_{\varepsilon} * \partial_{x}^{\alpha} g+\rho_{\varepsilon} * \partial_{x}^{\alpha} G_{1}+\rho_{\varepsilon} * \partial_{x}^{\alpha} G_{2}\right] d s, \quad t \geqq 0 .
\end{aligned}
$$

We take the $L^{8 / 3}\left(t, \infty ; L^{4}\right)$ norm of (3.68) to obtain by Lemma 2.1 (ii) with $q=4$ and $r=8 / 3$,

$$
\begin{aligned}
\left(\int_{t}^{\infty}\left\|\partial_{x}^{\alpha} z_{\varepsilon \lambda}(s)\right\|_{L^{4}}^{8 / 3} d s\right)^{3 / 8} \leqq & 2 K_{1} \int_{t}^{\infty}\left\|\partial_{x}^{\alpha}\left\{\left(A_{+}+R+v\right) \cdot \nabla\left(\rho_{\varepsilon} * z_{\varepsilon \lambda}\right)\right\}\right\|_{L^{2}} d s \\
& +K_{1} \int_{t}^{\infty}\left\|\partial_{x}^{\alpha} g\right\|_{L^{2}} d s+K_{1} \int_{t}^{\infty}\left\|\partial_{x}^{\alpha} G_{1}\right\|_{L^{2}} d s \\
& +K_{1} \int_{t}^{\infty}\left\|\partial_{x}^{\alpha} G_{2}\right\|_{L^{2}} d s, \quad t \geqq 0,
\end{aligned}
$$


where $K_{1}$ is defined in Lemma 2.1(ii). The summation of (3.69) over $|\alpha| \leqq 2$ gives us

$$
\begin{aligned}
& \sum_{|\alpha| \leqq 2}\left(\int_{t}^{\infty}\left\|\partial_{x}^{\alpha} z_{\varepsilon \lambda}(s)\right\|_{L^{4}}^{8 / 3} d s\right)^{3 / 8} \\
& \leqq \\
& \quad K_{1} \int_{t}^{\infty} \sum_{|\alpha| \leqq 2}\left\|\partial_{x}^{\alpha}\left\{\left(A_{+}+R+v\right) \cdot \nabla\left(\rho_{\varepsilon} * z_{\varepsilon \lambda}\right)\right\}\right\|_{L^{2}} d s \\
& \quad+K_{1} \int_{t}^{\infty} \sum_{|\alpha| \leqq 2}\left\|\partial_{x}^{\alpha} g\right\|_{L^{2}} d s+K_{1} \int_{t}^{\infty} \sum_{|\alpha| \leqq 2}\left\|\partial_{x}^{\alpha} G_{1}\right\|_{L^{2}} d s \\
& \quad+K_{1} \int_{t}^{\infty} \sum_{|\alpha| \leqq 2}\left\|\partial_{x}^{\alpha} G_{2}\right\|_{L^{2}} d s, \quad t \geqq 0 .
\end{aligned}
$$

Lemmas 2.2(i), 2.3, (3.54), (3.64) and the Gagliardo-Nirenberg inequality yield

$$
\begin{aligned}
\sum_{|x| \leqq 2}\left\|\partial_{x}^{\alpha}\left\{\left(A_{+}+R+v\right) \cdot \nabla\left(\rho_{\varepsilon} * z_{\varepsilon \lambda}\right)\right\}(s)\right\|_{L^{2}} & \\
\leqq & C\left[\left(\left\|A_{+}\right\| L_{L^{\infty}}+\|R\|_{L^{\infty}}+\|v\|_{L^{\infty}}\right)\left\|z_{\varepsilon \dot{\lambda}}\right\|_{H^{3}}\right. \\
& +\sum_{|\alpha|=1}\left(\left\|\partial_{x}^{\alpha} A_{+}\right\|_{L^{\infty}}+\left\|\partial_{x}^{\alpha} R\right\|_{L^{\infty}}+\left\|\partial_{x}^{\alpha} v\right\|_{L^{\infty}}\right)\left\|z_{\varepsilon \lambda}\right\|_{H^{2}} \\
& +\sum_{|\alpha|=2}\left(\left\|\partial_{x}^{\alpha} A_{+}\right\|_{L^{\infty}}+\left\|\partial_{x}^{\alpha} R\right\|_{L^{\infty}}\right)\left\|z_{\varepsilon \dot{\lambda}}\right\|_{H^{1}} \\
& \left.+\sum_{|\alpha|=2}\left\|\partial_{x}^{\alpha} v\right\|_{L^{4}}\left\|z_{\varepsilon \lambda}\right\|_{W^{1,4}}\right] \\
\leqq & C(1+\eta) \eta^{2}(1+s)^{-2}(\log (2+s))^{2}, \quad s \geqq 0
\end{aligned}
$$

By (3.70), (3.71), (3.12), (3.39), (3.41) and Lemma 2.6 we have

$$
\begin{aligned}
\sum_{|\alpha| \leqq 2}\left(\int_{t}^{\infty}\left\|\partial_{x}^{\alpha} z_{\varepsilon \lambda}(s)\right\|_{L^{4}}^{8 / 3} d s\right)^{3 / 8} & \\
\leqq & C K_{1}(1+\eta) \eta^{2} \int_{t}^{\infty}(1+s)^{-2}(\log (2+s))^{2} d s \\
& +K_{1} M \eta \int_{t}^{\infty}(1+s)^{-2}(\log (2+s))^{2} d s \\
& +C K_{1}(1+\eta)^{3} \eta^{2} \int_{t}^{\infty}(1+s)^{-2}(\log (2+s))^{2} d s \\
& +K_{1}(1+\eta)^{4} \eta^{3} \int_{t}^{\infty}(1+s)^{-2}(\log (2+s))^{2} d s \\
\leqq & \left\{5 K_{1} M \eta+C_{8}(1+\eta)^{5} \eta\left(K_{1} \eta\right)\right\}(1+t)^{-1}(\log (2+t))^{2}, \quad t \geqq 0
\end{aligned}
$$

where $C_{8}$ does not depend on $\varepsilon$ and $\lambda$. If we choose $\eta>0$ so small that

$$
C_{8}(1+\eta)^{5} \eta \leqq M
$$


then we obtain by (3.71)

$$
\sup _{t \geqq 0}\left[(1+t)(\log (2+t))^{-2} \sum_{|\alpha| \leqq 2}\left(\int_{t}^{\infty}\left\|\partial_{x}^{\alpha} z_{\varepsilon \lambda}(s)\right\|_{L^{4}}^{8 / 3} d s\right)^{3 / 8}\right] \leqq 6 K_{1} M \eta .
$$

When we consider passage to the limit as $\varepsilon \rightarrow+0$ and $\lambda \rightarrow+0,(3.54),(3.62)$, (3.66), (3.74), a compactness argument and the regularity theorem of linear theory show the existence of the solution $z(t)$ to (3.15)-(3.16) such that

$$
\begin{gathered}
z(t) \in \bigcap_{j=0}^{1} C^{j}\left([0, \infty) ; H^{3-2 j}\right), \\
\sup _{t \geqq 0}\left[(1+t)(\log (2+t))^{-2}\left(\sum_{|\alpha| \leqq 2}\left\|\partial_{x}^{\alpha} z(t)\right\|_{L^{2}}^{2}\right)^{1 / 2}\right] \leqq 6 M \eta, \\
\sup _{t \geqq 0}\left[(1+t)(\log (2+t))^{-2}\left(\sum_{|\alpha| \leqq 1}\left\|\partial_{x}^{\alpha} \partial_{t} z(t)\right\|_{L^{2}}^{2}\right)^{1 / 2}\right] \leqq 21 M \eta, \\
\sup _{t \geqq 0}\left[(1+t)(\log (2+t))^{-2}\left(\sum_{|\alpha|=1}\left\|\Delta \partial_{x}^{\alpha} z(t)\right\|_{L^{2}}^{2}\right)^{1 / 2}\right] \leqq 3(14 \sqrt{3}+1) M \eta, \\
\sup _{t \geqq 0}\left[(1+t)(\log (2+t))^{-2} \sum_{|x| \leqq 2}\left(\int_{t}^{\infty}\left\|\partial_{x}^{\alpha} z(s)\right\|_{L^{4}}^{8 / 3} d s\right)^{3 / 8}\right] \leqq 6 K_{1} M \eta
\end{gathered}
$$

We now prove the uniqueness of the solution $z(t)$ satisfying (3.75)-(3.79). We suppose that $z_{1}(t)$ and $z_{2}(t)$ are two solutions of (3.15)-(3.16) satisfying (3.75)-(3.79). If we put $Z(t)=z_{1}(t)-z_{2}(t)$, then $Z(t)$ satisfies (3.15) with $g(t) \equiv G_{1}(t, v, u)$ $\equiv G_{2}(t, v, u) \equiv 0$. Accordingly, in the same way as the case of the regularized problem we can prove that if $\eta>0$ is chosen so small that (3.53) holds, $Z(t)$ satisfies (3.54) with $4 M \eta$ replaced by zero. This implies the uniqueness.

Thus, if we choose $\eta>0$ so small that (3.10), (3.28), (3.31), (3.53), (3.60), (3.61), (3.64), (3.65) and (3.73) hold, then (3.34)-(3.38) and (3.75)-(3.79) show that $N[v, u]$ is a mapping from $X$ to $X$. In the same way as above, we can prove that if $\eta>0$ is chosen sufficiently small, then

$$
d\left(N\left[v_{1}, u_{1}\right], N\left[v_{2}, u_{2}\right]\right) \leqq \frac{1}{2} d\left(\left[v_{1}, u_{1}\right],\left[v_{2}, u_{2}\right]\right), \quad\left[v_{1}, u_{1}\right],\left[v_{2}, u_{2}\right] \in X
$$

Consequently, we conclude that if $\eta>0$ is sufficiently small, then $N[v, u]$ is a contraction mapping from $X$ to $X$. Therefore, there exists a unique fixed point $[v, u] \in X$ such that $[v, u]=N[v, u]$. Since $[v, u]$ belongs to the image of $N, v$ and $u$ must satisfy (3.34)-(3.38) and (3.75)-(3.79), respectively. Furthermore, by Corollary 2.5(i) and the definition of the mapping $N$ we can conclude that if we define $A(t)$ and $\psi(t)$ as in (3.1) and (3.2), respectively, then $A(t)$ and $\psi(t)$ are the solutions of (1.14)-(1.15) satisfying (1.5)-(1.9).

We next show that $A(t)$ satisfies the Coulomb gauge condition (1.3). We first note that $(4 \pi|x|)^{-1}$ is the fundamental solution of $-\Delta$. The application of the divergence operator to (1.14) yields

$$
\partial_{t}^{2}(\operatorname{div} A)-\Delta(\operatorname{div} A)=0, \quad t>0, \quad x \in \mathbf{R}^{3} .
$$


Since $\operatorname{div} A$ is a solution of (3.80), div satisfies the energy identity:

$$
\begin{aligned}
\left\|\partial_{t} \operatorname{div} A(t)\right\|_{L^{2}}^{2}+\|\nabla \operatorname{div} A(t)\|_{L^{2}}^{2}= & \left\|\partial_{t} \operatorname{div} A(s)\right\|_{L^{2}}^{2}+\|\nabla \operatorname{div} A(s)\|_{L^{2}}^{2} \\
= & \| \partial_{t} \operatorname{div} A_{+}(s)+\partial_{t} \operatorname{div} R(s) \\
& +\left\{\partial_{t} \operatorname{div} A(s)-\partial_{t} \operatorname{div} A_{+}(s)-\partial_{t} \operatorname{div} R(s)\right\} \|_{L^{2}}^{2} \\
& +\| \nabla \operatorname{div} A_{+}(s)+\nabla \operatorname{div} R(s) \\
& +\left\{\nabla \operatorname{div} A(s)-\nabla \operatorname{div} A_{+}(s)\right. \\
& -\nabla \operatorname{div} R(s)\} \|_{L^{2}}^{2}, \quad 0<t, s<\infty
\end{aligned}
$$

Letting $s \rightarrow \infty$ in (3.81), we have by (1.8), (2.31) and (1.13)

$$
\left\|\partial_{t} \operatorname{div} A(t)\right\|_{L^{2}}^{2}+\|\nabla \operatorname{div} A(t)\|_{L^{2}}^{2}=0, \quad t \geqq 0,
$$

which implies that $A(t)$ satisfies the Coulomb gauge condition (1.3). We can easily verify that (1.1)-(1.2) are equivalent to (1.14)-(1.15) for the solutions in the class of (1.5)-(1.7). Therefore, we conclude that $(A(t), \psi(t))$ are the solutions of $(1.1)-(1.3)$ satisfying (1.5)-(1.9).

It remains only to prove the uniqueness of the solutions satisfying (1.5)-(1.9). We omit the proof of the uniqueness, because the uniqueness follows from a similar (but slightly simpler) argument to above. For the details, see [13] and [14].

Concluding Remarks. (i) In the proof of Theorem 1.1 we have not used the gauge condition (1.3) at all, although the use of (1.3) makes the proof a little simpler. This is because we intend to make it clear that the gauge condition (1.3) does not play any essential role in the proof of Theorem 1.1. But the gauge condition plays an important role in the paper [5], which treats the initial value problem of the Maxwell-Dirac equations. It seems important to investigate the relation between the Maxwell-Schrödinger equations and the gauge condition.

(ii) The global existence of small amplitude solutions for the initial value problem of the covariant nonlinear wave equation has been studied through the $L^{\infty}-L^{2}$ estimates with weights related to the generators of the Poincare group (see, e.g., [1, 5 and 10]). Recently in [7], Hayashi has proved the global existence of small amplitude solutions for the initial value problem of the Schrödinger equation with quadratic nonlinearity by using the $L^{\infty}-L^{2}$ estimates with weights related to the generators of the Schrödinger group. It is conjectured that the global existence of small amplitude solutions for the initial value problem of the MaxwellSchrödinger equations could be proved through the $L^{\infty}-L^{2}$ estimates with weights related to the generators of the Poincare group and the Schrödinger group, although the Poincaré group and the Schrödinger group are not necessarily compatible.

\section{References}

1. Bachelot, A.: Problème de Cauchy globale pour des systèmes de Dirac-Klein-Gordon. Ann. Inst. Henri Poincaré, Physique Théorique 48, 387-422 (1988)

2. Bergh, J., Löfström, J.: Interpolation spaces. Berlin Heidelberg New York: Springer 1976

3. Flato, M., Simon, J., Taflin, E.: On global solutions of the Maxwell-Dirac equations. Commun. Math. Phys. 112, 21-49 (1987) 
4. Friedman, A.: Partial Differential Equations. New York: Holt-Rinehart and Winston, 1969

5. Georgiev, V.: Small amplitude solutions of the Maxwell-Dirac equations. Indiana Univ. Math. J. 4, 845-883 (1991)

6. Ginibre, J., Velo, G.: Scattering theory in the energy space for a class of nonlinear Schrödinger equations. J. Math. Pures Appl. 64, 363-401 (1985)

7. Hayashi, N.: Global existence of small solutions to quadratic nonlinear Schrödinger equations. (preprint)

8. Hayashi, N., Ozawa, T.: Modified wave operator for the derivative nonlinear Schrödinger equation. (preprint)

9. Klainerman, S.: Global existence for nonlinear wave equations. Commun. Pure Appl. Math. 33, 43-101 (1980)

10. Klainerman, S.: The null condition and global existence to nonlinear wave equation. Lect. in Appl. Math. 23, 293-326 (1986)

11. Nakamitsu, K., Tsutsumi, M.: Global existence of solutions to the Cauchy problem for coupled Maxwell-Schrödinger equations in two space dimensions. In: Physical Mathematics and Nonlinear Partial Differential Equations. Light-bourne, J.H., Rankin, S.M. (eds.), New York: Marcel Dekker 1988

12. Nakamitsu, K., Tsutsumi, M.: The Cauchy problem for the coupled Maxwell-Schrödinger equations. J. Math. Phys. 27, 211-216 (1986)

13. Ozawa, T.: Long range scattering for nonlinear Schrödinger equations in one space dimension. Commun. Math. Phys. 139, 479-493 (1991)

14. Ozawa, T., Tsutsumi, Y.: A symptotic behavior of solutions for the coupled Klein-GordonSchrödinger equations. (preptint)

15. Reed, M., Simon, B.: Methods of Modern Mathematical Physics III: Scattering Theory. New York: Academic Press, 1979

16. Stein, E.M.: Singular integrals and differentiability properties of functions. Princeton, NJ: Princeton University Press, 1970

17. Yajima, K.: Existence of solutions for Schrödinger evolution equations. Commun. Math. Phys. 110, 415-426 (1987)

Communicated by $\mathrm{H}$. Araki 